\title{
GALAXY-SCALE STAR FORMATION ON THE RED SEQUENCE: THE CONTINUED GROWTH OF SOs AND THE QUIESCENCE OF ELLIPTICALS
}

\author{
Samir Salim ${ }^{1}$, Jerome J. Fang ${ }^{2}$, R. Michael Rich $^{3}$, S. M. Faber ${ }^{2}$, and David A. Thilker ${ }^{4}$ \\ ${ }^{1}$ Department of Astronomy, Indiana University, Bloomington, IN 47404, USA; salims @ indiana.edu \\ ${ }^{2}$ University of California Observatories/Lick Observatory, University of California, Santa Cruz, CA 95064, USA \\ ${ }^{3}$ Department of Physics and Astronomy, University of California, Los Angeles, CA 90095, USA \\ ${ }^{4}$ Center for Astrophysical Sciences, The Johns Hopkins University, Baltimore, MD 21218, USA \\ Received 2011 December 28; accepted 2012 June 22; published 2012 August 1
}

\begin{abstract}
This paper examines star formation (SF) in relatively massive, primarily early-type galaxies (ETGs) at $z \sim 0.1$. A sample is drawn from bulge-dominated Galaxy Evolution Explorer/Sloan Digital Sky Survey (GALEX/SDSS) galaxies on the optical red sequence with strong UV excess and yet quiescent SDSS spectra. High-resolution far-UV imaging of 27 such ETGs using Hubble Space Telescope Advanced Camera for Surveys/Solar Blind Channel (ACS/SBC) reveals structured UV morphology in $93 \%$ of the sample, consistent with low-level ongoing $\mathrm{SF}\left(\sim 0.5 M_{\odot} \mathrm{yr}^{-1}\right)$. In $3 / 4$ of the sample the SF is extended on galaxy scales $(25-75 \mathrm{kpc})$, while the rest contains smaller (5-15 kpc) SF patches in the vicinity of an ETG-presumably gas-rich satellites being disrupted. Optical imaging reveals that all ETGs with galaxy-scale SF in our sample have old stellar disks (mostly S0 type). None is classified as a true elliptical. In our sample, galaxy-scale SF takes the form of UV rings of varying sizes and morphologies. For the majority of such objects we conclude that the gas needed to fuel current SF has been accreted from the intergalactic medium, probably in a prolonged, quasi-static manner, leading in some cases to additional disk buildup. The remaining ETGs with galaxy-scale SF have UV and optical morphologies consistent with minor merger-driven SF or with the final stages of SF in fading spirals. Our analysis excludes that all recent SF on the red sequence resulted from gas-rich mergers. We find further evidence that galaxy-scale SF is almost exclusively an S0 phenomenon ( $\sim 20 \%$ S0s have SF) by examining the overall optically red SDSS ETGs. Conclusion is that significant number of field S0s maintain or resume low-level SF because the preventive feedback is not in place or is intermittent. True ellipticals, on the other hand, stay entirely quiescent even in the field.
\end{abstract}

Key words: galaxies: elliptical and lenticular, $\mathrm{cD}$ - galaxies: evolution - ultraviolet: galaxies

Online-only material: color figures

\section{INTRODUCTION}

The processes that control global star formation (SF) in galaxies are at the core of many current studies that aim to understand galaxy formation and evolution. Efforts are being made both to recognize a range of relevant processes and, perhaps more challenging, to establish which processes dominate in different types of galaxies, in different environments, and at different cosmic epochs. Since at the present time the galaxies are still growing and transforming, many of the mechanisms of SF regulation can be studied at lower redshifts where the data are of much higher quality. However, despite major advances in this area, there are still many open questions.

One of the most enduring puzzles is presented by the evolution of massive early-type galaxies (ETGs), which comprise elliptical and lenticular (S0) galaxies. Their overall old stellar populations (e.g., Trager et al. 2000; De Lucia et al. 2006) and consequently the lack of current SF are to first order at odds with the otherwise successful model of hierarchical assembly of galaxies (Kauffmann et al. 1996) in which the massive galaxies formed most recently. While their morphological and kinematical transformation from disk galaxies into spheroid-dominated ones is very well explained by dissipative major mergers (especially for massive ellipticals; Barnes \& Hernquist 1996), the question of their subsequent quiescence is a separate question, and has started to be addressed only more recently with the introduction of various non-stellar feedback processes, especially the feedback from active galactic nuclei (AGNs; Springel et al. 2005). It now appears that in addition to shutting down of SF more or less concurrently with the morphological transformation, another feedback mechanism is required to keep an ETG free from subsequent SF (Croton et al. 2006). This requirement for a maintenance (or preventive) feedback is especially strong for field ETGs, which could be expected to continue accreting cold gas from the intergalactic environment (e.g., Gabor et al. 2011). Such accretion is probably the primary source of gas for actively star-forming galaxies as well (Kereš et al. 2005), without which they would not be able to sustain their observed star formation rates (SFRs) for longer than a few Gyr (Larson et al. 1980; Kennicutt et al. 1994; Bauermeister et al. 2010).

While ETGs are mostly quiescent almost by definition, the question is: are they entirely quiescent? What fraction is? If they exhibit SF, is it a new episode due to a fresh supply of gas, i.e., is galaxy being "rejuvenated," or are we seeing remnants of the original disk SF? Is, in the former case, the SF present because of the failure or absence of preventive feedback mechanism? Does $\mathrm{SF}$ tend to be widespread, as in spiral galaxies, or confined to circumnuclear regions? The dominance of old populations in ETGs makes the detection of relatively small amounts of SF intrinsically difficult. However, if SF could be systematically detected in ETGs, or, more generally, on the "red sequence," it would represent a potentially powerful way of identifying processes that lead to or prevent SF. In contrast, these regulative processes are more difficult to study in actively star-forming ("blue cloud") galaxies because of the high "background" of normal SF.

The presence of SF, especially in the central regions, has been firmly established in some nearby ETGs; e.g., in the SAURON 
survey of 48 S0s and ellipticals (Combes et al. 2007; Temi et al. 2009; Shapiro et al. 2010; Crocker et al. 2011). SF on galaxy scales, which we refer to as the extended SF, has received less attention, to some extent because of the difficulties in detecting it using optical methods. Detection of ionized emission can be challenging in ETGs where the strong continuum from old stars lowers the equivalent widths. Also, ionized emission can arise from a number of sources not associated with SF (Sarzi et al. 2010). Mid-IR emission (e.g., $24 \mu \mathrm{m}$ from Spitzer/MIPS) in ETGs can have an order of magnitude stronger contribution from intermediate-age and older stellar populations than from the young stars (Salim et al. 2009; Kelson \& Holden 2010). The most promising method is the UV emission from young massive stars, which is an order of magnitude more sensitive to recent SF than the blue optical flux (Kauffmann et al. 2007), and probes timescales that are closer to the current SF than those probed by the blue light from less massive, longer lived stars $(\sim 100 \mathrm{Myr}$ versus $\sim 1$ Gyr). ${ }^{5}$

Large-scale detection and characterization of ETGs in the UV was facilitated with the UV surveys of Galaxy Evolution Explorer (GALEX; Martin et al. 2005). Initial GALEX studies (e.g., Yi et al. 2005; Rich et al. 2005) based their approach on selecting large statistical samples of galaxies on the red optical sequence, which is where most ETGs are found. While most optical red galaxies remained red in the UV-optical colors, a significant fraction exhibited a UV excess, which Yi et al. (2005) interpreted as low levels of ongoing SF. ${ }^{6}$ Since these studies culled their samples from Sloan Digital Sky Survey (SDSS) spectroscopic survey in which galaxies are typically found at $z \sim 0.1$, little could be said about the morphology of the UV light (GALEX has a resolution of $\left.5^{\prime \prime}\right)$ and consequently whether this UV excess actually arose from young stars.

Subsequent efforts focused on the UV morphology to confirm the presence of the extended SF in the optical red sequence and/or among individual ETGs (Donovan et al. 2009; Thilker et al. 2010; Cortese \& Hughes 2009; Salim \& Rich 2010; Marino et al. 2011; Lemonias et al. 2011). However, many questions remained: what is the origin of the star-forming gas in ETGs?; has this SF started recently or is it prolonged?; is SF related to the processes of disk building?; and how does the SF relate to the two types of ETGs: lenticulars (SOs) and ellipticals (Es)? This paper and the accompanying work (Fang et al. 2012) address these questions using a sample of 29 ETGs selected from SDSS and GALEX surveys for which detailed far-UV images were obtained with the Hubble Space Telescope (HST). The initial analysis of this sample was presented in Salim \& Rich (2010, hereafter SR2010), where it was shown that these ETGs, selected on the basis of the presence of a strong UV excess, exhibit clear signatures of extended SF. The current work expands on the morphological and size-related aspects of the analysis of the $H S T$ sample and appends it with the analyses of the general population of ETGs from SDSS and GALEX. Paper II tackles SF histories of this sample using surface brightness photometry, and also discusses the selection of more complete samples of ETGs with extended SF.

\footnotetext{
5 We note that far-UV (FUV; $\lambda \sim 1500 \AA$ ) is easier to interpret as SF than the longer wavelength near-UV (NUV; $\lambda \sim 2300 \AA$ ), which in ETGs primarily comes from main-sequence turnoff stars and is strongly dependent on the stellar metallicity (Donas et al. 2007; Dorman et al. 2003; Smith et al. 2012).

6 This underlines the importance of specifying the type of color (optical versus UV-optical) when referring to the red sequence, as the optical red sequence will contain both truly quiescent galaxies and those with small relative amounts of $\mathrm{SF}$.
}

The paper is structured as follows. Selection of the HST sample is explained in Section 2, and the resulting UV observations and optical imaging data from SDSS and WIYN are described in Section 3. Results are given in the subsequent five sections. In Sections 4, 5, and 6, we provide morphological analysis based on the UV, $\mathrm{H} \alpha$, and optical imaging, respectively, which shows that the majority of the sample has SF on large scales (tens of $\mathrm{kpc}$ ), and that the SF is found exclusively in S0s and not in ellipticals. This is followed by two sections that place our sample in context. In Section 7, we analyze the relation between the UV and optical morphologies, showing that the star-forming gas is preferentially acquired subsequent to a galaxy getting onto a red sequence, while in Section 9 we further explore differences between S0 and elliptical galaxies in terms of SF and determine the incidence rate of SF in the two types in the overall population. Discussion of the results in view of evolutionary scenarios is given in Section 10, and the findings are summarized in Section 11. Cosmology parameters $\Omega_{m}=0.3, \Omega_{\Lambda}=0.7$, $H_{0}=70 \mathrm{~km} \mathrm{~s}^{-1} \mathrm{Mpc}^{-1}$ are assumed throughout.

\section{SAMPLE SELECTION}

The sample presented in this paper comprises 29 galaxies selected from SDSS and GALEX catalogs and observed with the Solar Blind Channel (SBC), a UV detector of the Advanced Camera for Surveys (ACS) on board the HST. These observations were taken in program GO-11158 (PI: Rich). No other observations with HST instruments were carried out. While 30 galaxies were originally selected to be observed with the HST, one target was not successfully imaged. We refer to the resulting 29 as the $H S T$ sample. Here we provide a more detailed description of the sample selection presented in SR2010.

1. SR2010 started with 67,883 galaxies from the main spectroscopic survey of SDSS DR4 that lie within the $645 \mathrm{deg}^{2}$ overlap with GALEX Medium-Imaging Survey (internal data release IR1.1), regardless of whether the galaxies are detected by GALEX or not. Additional details about this parent sample are given in Salim et al. (2007).

2. To obtain galaxies with larger angular sizes and better UV measurements, the selection was restricted to $z<0.12$, which left 30,860 galaxies.

3. Both far-UV (FUV) and near-UV (NUV) detections were required, plus GALEX detections were required not to fall close to the edge of the circular detector $(<0.55 \mathrm{deg})$. Asking for NUV was meant to eliminate any spurious FUVonly detections. These cuts brought the sample to 17,574 galaxies.

4. Next, SR2010 required that the optical light profile be consistent with an ETG, by asking for an SDSS concentration (the ratio of the radii enclosing $90 \%$ and $50 \%$ of the Petrosian flux in $r$ band $^{7}$ ) to be $C>2.5$ (Strateva et al. 2001; Bernardi et al. 2003). Such a cut yields a very complete sample of ETGs, while allowing non-negligible latetype contamination (Bernardi et al. 2010; Masters et al. 2010b), which was removed by subsequent cuts. This step left 7096 galaxies.

5. (a) In order to select only galaxies with no spectroscopic signs of ongoing SF or nuclear activity, SR2010 used the emission-line classification of Brinchmann et al. (2004),

\footnotetext{
In SR2010 the concentration was erroneously reported to be based on $i$ band. The difference in the two measures is negligible.
} 
applied to SDSS DR4 by MPA/JHU group, and accepted only what B04 call "unclassifiable" galaxies, which is equivalent to a cut on $\mathrm{H} \alpha$ flux signal-to-noise ratio $(\mathrm{S} / \mathrm{N})$ of $\lesssim 3$. This resulted in 1660 galaxies. (b) While the previous cut should have removed most Type 2 AGNs, it was noticed that the scaling of line flux errors suggested by the MPA/JHU group to reproduce systematic errors leads to the overestimation of line ratio errors (because the systematic errors of lines that are close to each other in wavelength are correlated), so additional galaxies were excluded if the line ratios would place them in the LINER region of the BPT diagram $\left(-0.2 \leqslant \log \left(\left[\mathrm{N}_{\mathrm{II}}\right] / \mathrm{H} \alpha\right)<0.5\right.$ and $-0.3 \leqslant$ $\log ([\mathrm{O} \mathrm{III}] / \mathrm{H} \beta)<0.8)$, regardless of line $\mathrm{S} / \mathrm{N}$. This cut left 986 galaxies. (c) For several dozen of the remaining galaxies the formal $\mathrm{H} \alpha \mathrm{S} / \mathrm{N}$ is low simply because the part of the spectrum around $\mathrm{H} \alpha$ was corrupted. For them the $\mathrm{H} \alpha$ cut is replaced by asking that $\mathrm{H} \beta$ flux $\mathrm{S} / \mathrm{N}$ be $<3$. Removing these sources left 954 galaxies. This represents the sample of apparently quiescent ETGs.

6. From quiescent ETGs, SR2010 selected those with strong UV excess, $\sim 2 \times$ stronger than the maximum excess expected UV upturn from old populations. They defined this cut as rest-frame color FUV $-r<5.3$ (rest-frame colors derived using KCORRECT v4, Blanton \& Roweis 2007; all magnitudes in AB system). This resulted in 63 galaxies. This cut, like others, was not meant to produce a complete sample, but rather one with minimal contamination from late-type galaxies and a sample size that could be practically observed with the HST.

7. The final selection cut this number in half, to 30 , by applying visual inspection of SDSS postage stamp images (gri composites) and SDSS spectra. Most of the candidates were discarded because there was a nearby blue source that gave rise to the UV excess. Other reasons for exclusion include non-early-type interlopers (spiral arms or the absence of bulge), and the presence of E+A spectral features.

Most recent GALEX data processing (GR6) as well as the FUV measurements from the HST UV images themselves (Paper II) have revealed that two galaxies in the sample had greatly overestimated FUV magnitudes in the original (IR1.1) GALEX photometry (difference of $\sim 1$ and $\sim 2 \mathrm{mag}$ ). Their true magnitudes would not qualify these objects as having a strong UV excess, so we discuss them apart from the rest of the sample. This brings the total number of galaxies that have a strong UV excess and for which HST observations produced detections to 27 . We denote individual galaxies by a prefix SR, followed by a sequential number (01 to 30$)$, which indicates the rank in terms of FUV $-r$ color in the original list, with 01 being the bluest object. Note that HST failed to observe one galaxy, but we retain it in the numbering order (SR24).

The resulting sample has a general appearance of ETGs in SDSS gri color composites and red optical colors $(g-r>0.7$ rest frame, Figure 21) despite no explicit optical color selection. Such colors place the sample fully within the optical red sequence, yet the UV excess ensures that they occupy the intermediate color region of the UV-optical color-magnitude (mass) diagram (Figure 1), known as the "green valley" (Wyder et al. 2007). Furthermore, none of the galaxies in the resulting sample belongs to clusters from the GMBCG catalog of Hao et al. (2010) and its $z<0.1$ addendum.

In our selection, the general quiescence is ensured by removing galaxies with emission lines in SDSS spectra. This criterion also leads to a sample with heavily suppressed SF in the central

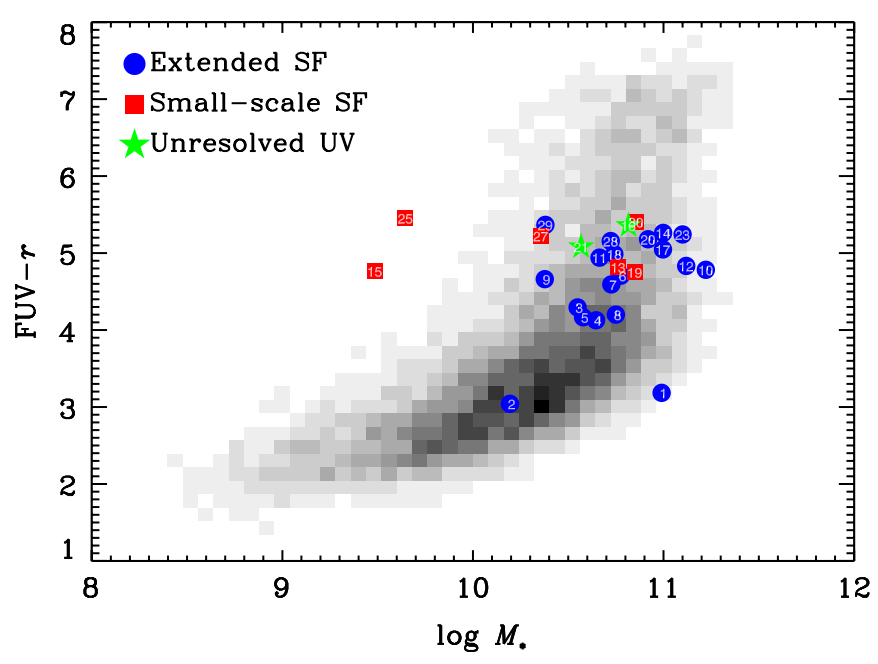

Figure 1. UV-to-optical color vs. stellar mass diagram. The HST sample of ETGs with strong UV excess is shown as colored symbols, split into UV morphology classes: extended SF (blue dots), small-scale SF (red squares), and unresolved (green stars). Numbers within symbols indicate object identifications (SR\#). Gray-scale image represents the distribution of the underlying galaxies detected by SDSS and GALEX. Truly quiescent ETGs are redder than the HST sample, while the actively star-forming galaxies ("blue sequence") are bluer than the HST sample. The sample occupies the region of intermediate colors known as the green valley.

(A color version of this figure is available in the online journal.)

( $\sim 5 \mathrm{kpc}$ ) region covered by SDSS fiber. Is there a significant population of ETGs with extended SF that is not covered by our selection, and is such population likely to exhibit very different UV morphology from that seen in our sample? We examine these issues in detail in the Appendix. We conclude that our $H S T$ sample covers $\sim 2 / 3$ of the parameter space occupied by ETGs with extended SF. What the HST sample does not cover are ETGs (still on the optical red sequence) in which the extended emission is concurrent with the SF in the central regions. Such galaxies may in some cases exhibit UV morphologies different from those in our HST sample; specifically there may be more in-filled disks than what we observe. Any implications of these selection effects will be discussed in appropriate sections.

\section{DATA AND OBSERVATIONS}

\subsection{HST UV Imaging}

HST ACS/SBC imaging of UV-strong ETGs represents the backbone of this project. The SBC is a multi-anode microchannel array with a parallelogram-shaped field of view of $\sim 35^{\prime \prime} \times 31^{\prime \prime}$ and $\sim 0^{\prime \prime}$.03 pixel $^{-1}$ scale. The FWHM is however $\sim 0^{\prime \prime}$, 3 , which is nevertheless an order of magnitude better that that of GALEX, allowing us to study the detailed spatial distribution of the UV emission at typical redshifts of SDSS galaxies $(z \sim 0.1)$. Imaging was performed using the F125LP long-pass filter $\left(\lambda_{\text {eff }}=1459 \AA\right)$, which has a similar effective wavelength as the GALEX FUV filter.

A four-point dither strategy was employed to improve the sampling, and the individual exposures were processed using MultiDrizzle tasks (Koekemoer et al. 2002). Each galaxy was observed for one orbit ( $\sim 2.5 \mathrm{ks}$ total, or $\sim 600 \mathrm{~s}$ per exposure). Below we describe in more detail the reduction process, which was modified from the pipeline reduction to address specific issues related to SBC data. 


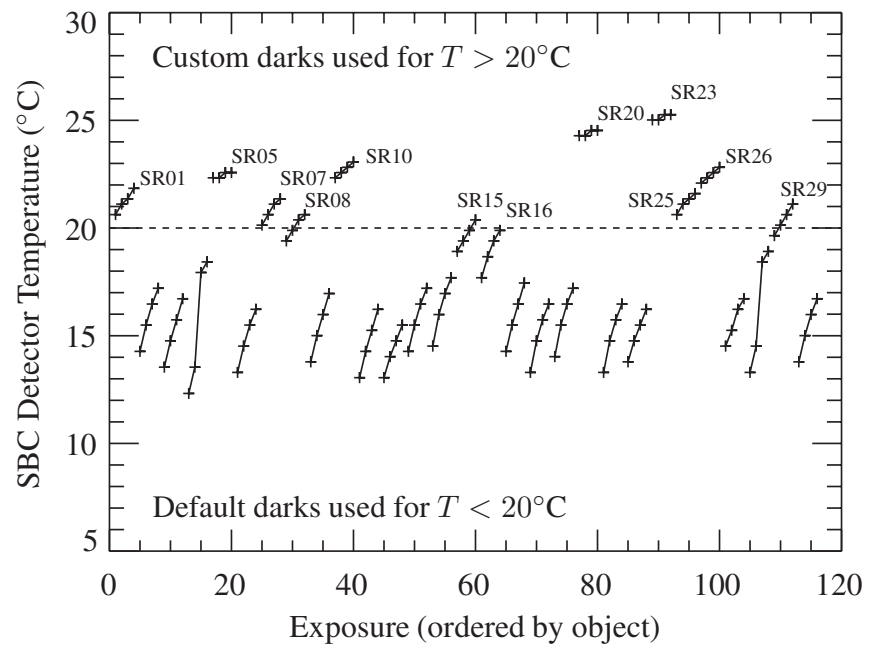

Figure 2. SBC detector temperature for each individual exposure. The temperature increases over the course of each four-exposure set (connected points). The pipeline-supplied dark files were used in reducing the raw exposures for which the detector temperature was cold $\left(T \lesssim 20^{\circ} \mathrm{C}\right)$. Exposures for which $T \gtrsim 20^{\circ} \mathrm{C}$ required the use of custom darks that accounted for the increased dark current. Galaxies for which custom darks were applied are labeled.

\subsubsection{SBC Dark Current Subtraction}

The SBC suffers from a temperature-dependent dark current that results in a steadily increasing "glow" near the center of the detector as it operates for extended periods (e.g., Teplitz et al. 2006), i.e., over the course of an orbit. Figure 2 shows the detector temperature (value extracted from the raw image FITS header keyword MDECODT1) for each exposure. While the majority of exposures were taken at $T<20^{\circ} \mathrm{C}$, several were obtained at $T>20^{\circ} \mathrm{C}$ and suffer from severe dark current. The master dark files provided by the data reduction pipeline only stack dark frames taken when the detector is cold, $T \lesssim 20^{\circ} \mathrm{C}$, and are thus inadequate for removing the increased dark current in exposures taken at higher temperatures. To accurately subtract the dark current in high-temperature frames we made custom dark images based on dark frames taken at $T>20^{\circ} \mathrm{C}$. Single dark exposures taken over a range of temperatures are available from the HST archive (see Cox 2009 for details). We constructed five custom dark images by stacking several individual dark frames covering the temperature range $20^{\circ} \mathrm{C}<T<25.5^{\circ} \mathrm{C}$ and smoothing the resulting stack. Each stack contains 5-8 dark frames spanning a narrow range in detector temperature.

The individual raw science frames were then processed using the dark image corresponding to the temperature of the detector at the time of exposure. In a few raw frames, the glow was not fully removed with the dark image corresponding to a temperature from FITS header, so for those a dark image corresponding to a higher temperature was used to completely remove the dark current glow. In these cases the choice of which dark to use was based on visually inspecting the resulting processed science image and choosing the dark image that best removed any residual dark current.

\subsubsection{Constructing ACS Co-added Images}

The final science image is a stack of the four individual exposures processed using MultiDrizzle, which corrects for geometrical distortion and shifts and registers each exposure onto a common coordinate system. In addition to dark current effects, SBC images exhibit time-variable "sky" background, which in some exposures dominates the signal. This background

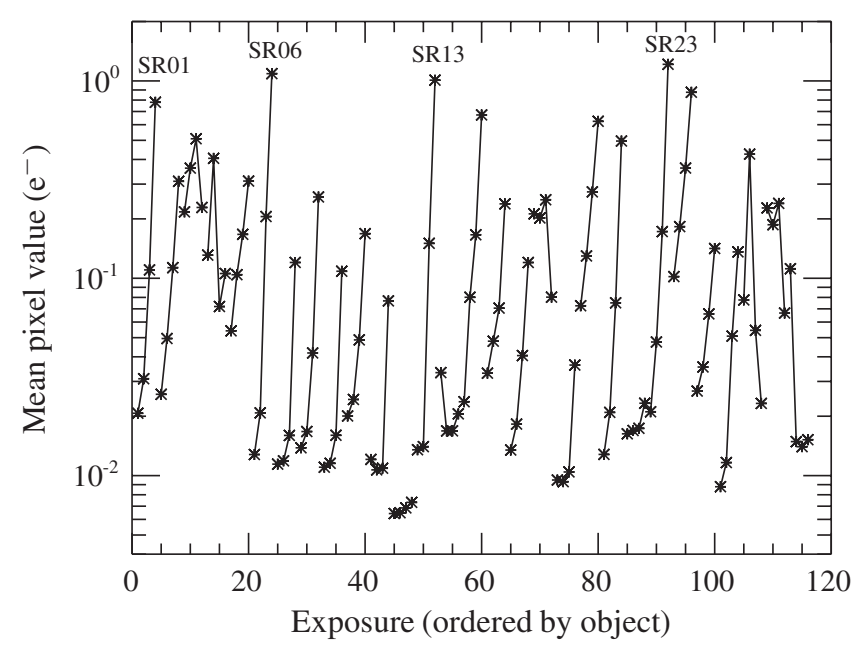

Figure 3. Mean pixel value for individual raw exposures after the dark current subtraction. For clarity, each set of four exposures (one orbit) is connected by a solid line. Four targets have been labeled to guide the eye. Nearly all objects show an increase in the mean pixel value over the course of four exposures, reflecting the increase in the detected geocoronal background over the course of each orbit.

comes from the geocoronal emission lines. While most of the geocoronal flux is in $\operatorname{Ly} \alpha$ and is therefore blocked by the choice of F125LP filter, some background also arises from O I at $1304 \AA$ (Maybhate et al. 2010). Geocoronal emission is quite variable. This is demonstrated in Figure 3, where we plot the mean pixel value in each raw exposure after masking out the object(s) in each. For each set of four exposures, the mean value varies dramatically (up to two orders of magnitude in some cases). Visual inspection of the exposures confirms that the increase in the mean is due to the sky background, which increasingly illuminates the exposures uniformly across the field of view, rather than any residual dark current glow, which is concentrated toward the center of each frame. Note that a large mean value does not necessarily correlate with a high dark current in the exposure (compare with Figure 2). Indeed, some exposures taken at cold temperatures have very large mean pixel values relative to the others. This reinforces the fact that the sky background is dominated by scattered light rather than by the thermal dark current of the detector. To account for the variable background, we weighted each image by the inverse of the mean pixel value when combining them in MultiDrizzle. ${ }^{8}$ In this way co-added images have greater contributions from exposures with lower sky background.

Since the data are dominated by Poisson statistics and most pixels receive no counts we finally apply smoothing with a Gaussian kernel of 10 pixel $(1 \sigma)$. This yields images with a point-spread function (PSF) FWHM of $\sim 0$.' 6 , comparable to the seeing in our optical images. Smoothing greatly improves our ability to see faint features while preserving genuine details. HST SBC imaging is presented in the first column of imaging panels in Figures 4-13.

\subsection{SDSS Optical Imaging}

One of the goals of this work is to compare the UV and optical morphologies of the HST sample. For optical morphologies we draw from two sources: SDSS ugriz imaging and our WIYN $3.5 \mathrm{~m} R$-band imaging. WIYN $3.5 \mathrm{~m}$ imaging is significantly deeper than that of SDSS and has a better spatial resolution.

\footnotetext{
8 Since the counts in SBC images have a Poisson distribution, variance is the
} same as the mean value. 


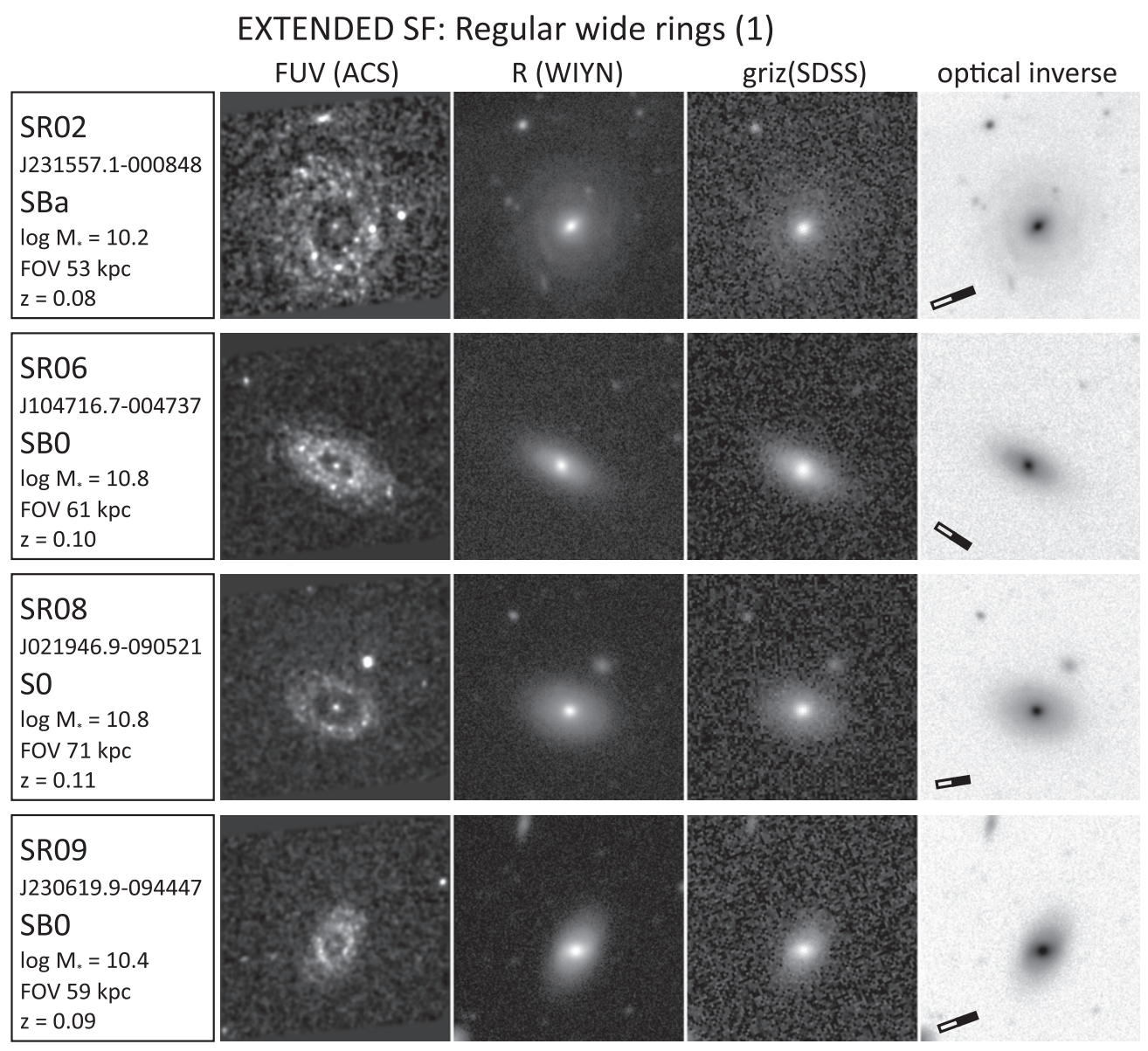

Figure 4. Far-UV and optical images of early-type galaxies with extended SF and regular wide UV rings (first set of two). Data box on the left gives galaxy designation (SR stands for Salim \& Rich 2010), SDSS ID based on J2000 coordinates, Hubble type, stellar mass, the extent of the horizontal field of view in kpc, and the redshift. All imaging panels span $35^{\prime \prime}$. First imaging panel shows the HST (ACS/SBC) far-UV image smoothed with a Gaussian kernel (FWHM $=0$ "'6). Second panel shows the deep ( $\sim 30$ minutes) $R$-band image obtained with the $3.5 \mathrm{~m}$ WIYN telescope (when available). Third panel displays the SDSS image constructed by co-adding griz exposures ( $\sim 4$ minutes). The last panel shows the inverted optical image from WIYN, if available, or SDSS otherwise. The scale bar shows $10 \mathrm{kpc}$ with the black end pointing toward north, with east being 90 deg counterclockwise. ACS/SBC field has a parallelogram shape, with the upper left and lower right slivers in rectangular panels not covered. Galaxies are not centered in ACS/SBC field, but are offset primarily downward. Pixel intensity range was chosen in such a way to avoid saturating the bright bulge. Linear scaling between the ADUs and pixel intensity was used for the FUV image, while optical images use asinh scaling (Lupton et al. 2004), which allows the full dynamic range to be presented. This leads to the smaller, unsaturated appearance of the bulges compared to the views that would be obtained using the linear scaling. The ordering of galaxies follows the SR numbering, which in turn goes from the bluest (in FUV $-r$ ) to the reddest.

However, it is not available for the entire sample and thus needs to be supplemented by SDSS images.

SDSS imaging is performed in five filters, and each is exposed for about $54 \mathrm{~s}$. Typical seeing is 1 ."4. To increase the effective depth of SDSS images we co-add four bands ( griz) together. $^{9}$ Since $u$ band is generally not sensitive enough to detect extended galaxy structure (e.g., S0 disks) at this redshift, we do not include it in the co-add. We show the co-added SDSS images of each galaxy in the third column of imaging panels in Figures 4-13. SDSS images are presented even when deeper WIYN images are available for two reasons. First, to help evaluate the extent of features that may not be detectable when only SDSS images are available, and second, to present images that are similar to those that were originally used to select the sample.

\subsection{WIYN 3.5 m Optical Imaging}

The optical imaging at the WIYN $3.5 \mathrm{~m}$ telescope focused on the $2 / 3$ of the sample having higher UV surface brightness

\footnotetext{
9 Co-adding of griz images was performed without any weighting, as it produced very similar results (in terms of perceived depth) as the more elaborate co-addition of scaled variance-weighted images in five filters, with
} scaling based on the total flux in a given band. as seen in the HST UV images. The imaging consisted of deep $R$-band observations accompanied, for a subset of objects, by narrowband $\mathrm{H} \alpha+[\mathrm{N}$ II] observations. The goal of deep $R$ imaging was to facilitate more accurate optical classification (including the presence of bars) and to reveal any signatures of interaction (such as tidal features, shells or dust lanes). Narrowband imaging had the goal of searching for $\mathrm{H} \alpha$ ionizing emission that would be indicative of ongoing SF occurring on timescales shorter than those probed by UV bright population. Analysis facilitated by these observations is presented in Sections 5 and 6.

Observing took place over two runs (UT 2010 Mar 22-24 and UT 2010 Aug 7-11). Out of 8 total nights, weather permitted observing on 3.5. As a result, 18 galaxies were observed in $R$ and 13 in narrow band. We used the MiniMo CCD camera with a pixel scale of 0 . $^{\prime} 14$. Median total exposure times per galaxy were 30 minutes in $R$ and 40 minutes in a narrow band (see Table 1). Individual exposure times were 10 minutes in $R$ and 20 minutes in the narrow bands. Median seeing was 0.8 in $R$ and 0.7 in narrow bands. We used the KPNO Combined Set of $\mathrm{H} \alpha$ narrowband filters, choosing one among seven filters with central wavelength closest to the wavelength of redshifted $\mathrm{H} \alpha$. Filters have an approximate FWHM of $80 \AA$. Scaled $R$-band 
Table 1

Properties of the HST Sample of ETGs with Strong UV Excess ${ }^{\mathrm{a}}$

\begin{tabular}{|c|c|c|c|c|c|c|c|c|c|c|c|c|}
\hline Name & IAU Designation & $\begin{array}{l}\text { R.A. (J2000) } \\
\text { (deg) }\end{array}$ & $\begin{array}{l}\text { Decl. (J2000) } \\
\text { (deg) }\end{array}$ & Redshift & $\log M_{*}$ & log SFR & $\begin{array}{l}t_{\exp }(R) \\
(\mathrm{s})\end{array}$ & $\begin{array}{c}t_{\exp }(\mathrm{NB}) \\
(\mathrm{s})\end{array}$ & $\begin{array}{l}\text { Hubble } \\
\text { Type }\end{array}$ & UV Class & UV Subclass & $\begin{array}{c}\text { UV Size } \\
(\mathrm{kpc})\end{array}$ \\
\hline SR01 & SDSS J204524.60-044634.2 & 311.352510 & -4.776174 & 0.119 & $10.99 \pm 0.09$ & $0.10 \pm 0.50$ & 3000 & $2400^{*}$ & S0/a & Extended SF & Irreg. ring $\mathrm{w} / \mathrm{extreme}$ disk & 75 \\
\hline SR02 & SDSS J231557.10-000848.8 & 348.987945 & -0.146896 & 0.081 & $10.19 \pm 0.06$ & $-0.58 \pm 0.04$ & 2400 & $2400^{\dagger}$ & $\mathrm{SBa}$ & Extended SF & Regular wide ring & 35 \\
\hline SR03 & SDSS J162021.48+491609.0 & 245.089529 & 49.269172 & 0.057 & $10.55 \pm 0.04$ & $-0.78 \pm 0.08$ & & & SB0 & Extended SF & Regular wide ring & 30 \\
\hline SR04 & SDSS J084612.82+522101.4 & 131.553432 & 52.350410 & 0.114 & $10.65 \pm 0.06$ & $-0.48 \pm 0.14$ & & 1200 & So & Extended SF & Narrow ring(s) & 31 \\
\hline SR05 & SDSS J080804.34+501256.0 & 122.018109 & 50.215561 & 0.101 & $10.58 \pm 0.07$ & $-0.09 \pm 0.19$ & 600 & 900 & SB0 & Extended SF & Narrow ring(s) & 26 \\
\hline SR06 & SDSS J104716.72-004737.9 & 161.819701 & -0.793885 & 0.095 & $10.78 \pm 0.07$ & $-0.27 \pm 0.18$ & 600 & 1200 & SB0 & Extended SF & Regular wide ring & 37 \\
\hline SR07 & SDSS J001156.72-104934.7 & 2.986350 & -10.826323 & 0.107 & $10.73 \pm 0.07$ & $-0.43 \pm 0.21$ & 1800 & & So & Extended SF & Irreg. ring $\mathrm{w} / \mathrm{extreme}$ disk & 59 \\
\hline SR08 & SDSS J021946.93-090521.1 & 34.945579 & -9.089211 & 0.111 & $10.75 \pm 0.07$ & $-0.48 \pm 0.17$ & 1200 & 2400 & So & Extended SF & Regular wide ring & 27 \\
\hline SR09 & SDSS J230619.97-094447.0 & 346.583222 & -9.746410 & 0.092 & $10.38 \pm 0.08$ & $-0.60 \pm 0.31$ & 1800 & & SB0 & Extended SF & Regular wide ring & 25 \\
\hline SR10 & SDSS J024041.36-084947.4 & 40.172352 & -8.829841 & 0.111 & $11.22 \pm 0.06$ & $0.09 \pm 0.17$ & 600 & & so & Extended SF & Disk w/hole & 42 \\
\hline SR11 & SDSS J140216.66+025902.7 & 210.569453 & 2.984093 & 0.077 & $10.66 \pm 0.09$ & $0.05 \pm 0.21$ & 600 & $2200^{\dagger}$ & SBab & Extended SF & Arms (pseudoring) & 34 \\
\hline SR12 & SDSS J014214.66-091103.6 & 25.561091 & -9.184346 & 0.114 & $11.12 \pm 0.07$ & $0.36 \pm 0.18$ & 1800 & $3600^{*}$ & $\mathrm{Sa}$ & Extended SF & Disk w/hole & 46 \\
\hline SR13 & SDSS J160830.21+534107.9 & 242.125866 & 53.685539 & 0.107 & $10.76 \pm 0.06$ & $-0.10 \pm 0.19$ & 1800 & & $\mathrm{E} / \mathrm{S} 0$ & Small-scale SF & Off-center & 9 \\
\hline SR14 & SDSS J161646.04+010255.4 & 244.191861 & 1.048746 & 0.085 & $11.00 \pm 0.08$ & $-0.07 \pm 0.17$ & 1800 & $2400^{*}$ & SB0/a & Extended SF & Disk w/hole & 34 \\
\hline SR15 & SDSS J101807.52-004857.7 & 154.531362 & -0.816040 & 0.033 & $9.49 \pm 0.09$ & $-1.58 \pm 0.37$ & & & E & Small-scale SF & Central & 5 \\
\hline SR16 & SDSS J041246.35-055129.4 & 63.193153 & -5.858179 & 0.100 & $10.82 \pm 0.08$ & $-0.54 \pm 0.46$ & & & S0 (pec) & Unresolved & & \\
\hline SR17 & SDSS J225131.65-102218.9 & 342.881890 & -10.371922 & 0.118 & $11.00 \pm 0.07$ & $-0.10 \pm 0.19$ & 2400 & $2400^{\dagger}$ & Sab & Extended SF & Arms (tidal) & 38 \\
\hline SR18 & SDSS J161041.32+512600.4 & 242.672188 & 51.433444 & 0.116 & $10.74 \pm 0.07$ & $-0.04 \pm 0.56$ & 1200 & & So & Extended SF & Regular wide ring & 34 \\
\hline SR19 & SDSS J002950.16-090833.5 & 7.459033 & -9.142660 & 0.118 & $10.85 \pm 0.07$ & $-0.47 \pm 0.20$ & & & E/S0 & Small-scale SF & Off-center & 11 \\
\hline SR20 & SDSS J084424.13+520122.0 & 131.100575 & 52.022785 & 0.114 & $10.92 \pm 0.07$ & $-0.04 \pm 0.22$ & & & So & Extended SF & Regular wide ring & 32 \\
\hline SR21 & SDSS J133743.93+030646.8 & 204.433061 & 3.113001 & 0.112 & $10.57 \pm 0.07$ & $-0.03 \pm 0.22$ & & & $\mathrm{E}$ & Unresolved & & \\
\hline SR22 & SDSS J041105.03-053412.8 & 62.770969 & -5.570247 & 0.066 & $10.56 \pm 0.07$ & $-2.46 \pm 0.65$ & & & E & No UV excess & & \\
\hline SR23 & SDSS J085208.61+552847.9 & 133.035874 & 55.480000 & 0.114 & $11.10 \pm 0.07$ & $-0.41 \pm 0.19$ & & & So & Extended SF & Regular wide ring & 34 \\
\hline SR25 & SDSS J084949.45+570713.7 & 132.456064 & 57.120499 & 0.042 & $9.65 \pm 0.08$ & $-2.08 \pm 0.69$ & & & $\mathrm{E}$ & Small-scale SF & Central & 4 \\
\hline SR26 & SDSS J030415.99-084057.1 & 46.066662 & -8.682551 & 0.117 & $10.87 \pm 0.07$ & $-0.44 \pm 0.27$ & & & $\mathrm{E} / \mathrm{S} 0$ & No UV excess & & 35 \\
\hline SR27 & SDSS J140540.19+034710.9 & 211.417487 & 3.786375 & 0.092 & $10.36 \pm 0.08$ & $-1.50 \pm 0.63$ & 600 & 2400 & $\mathrm{E} / \mathrm{S} 0$ & Small-scale SF & Off-center & 10 \\
\hline SR28 & SDSS J235959.36-111131.5 & 359.997370 & -11.192087 & 0.104 & $10.72 \pm 0.07$ & $-0.37 \pm 0.26$ & 1800 & & SB0 & Extended SF & Narrow ring(s) & 25 \\
\hline SR29 & SDSS J161521.62+491841.5 & 243.840122 & 49.311544 & 0.059 & $10.38 \pm 0.09$ & $-0.38 \pm 0.59$ & 2400 & 2400 & So & Extended SF & Narrow ring(s) & 12 \\
\hline SR30 & SDSS J143607.80+041507.4 & 219.032527 & 4.252061 & 0.114 & $10.86 \pm 0.07$ & $-0.69 \pm 0.27$ & 860 & 1950 & So & Small-scale SF & Off-center & 15 \\
\hline
\end{tabular}

Notes.

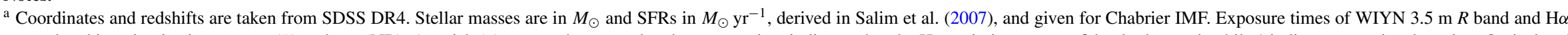

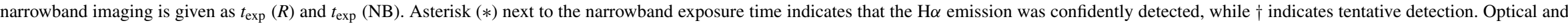

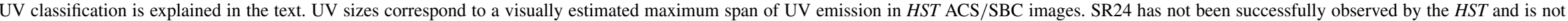
listed here. 


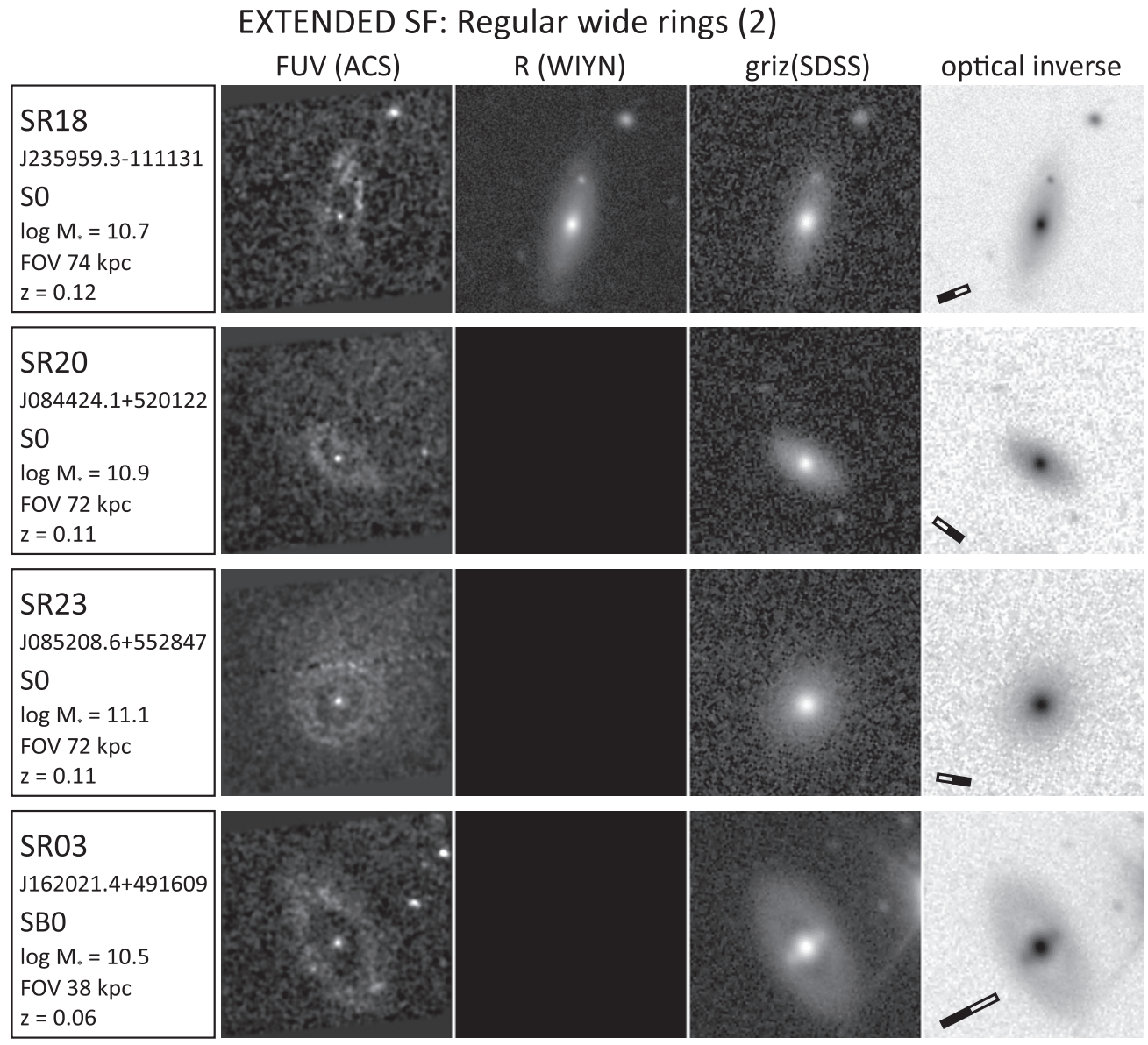

Figure 5. Far-UV and optical images of early-type galaxies with extended SF and regular wide UV rings (second set). See Figure 4 for full description. SR03 does not follow the ordering scheme and is placed at the end because of its unique optical appearance.

EXTENDED SF: Disks with small holes

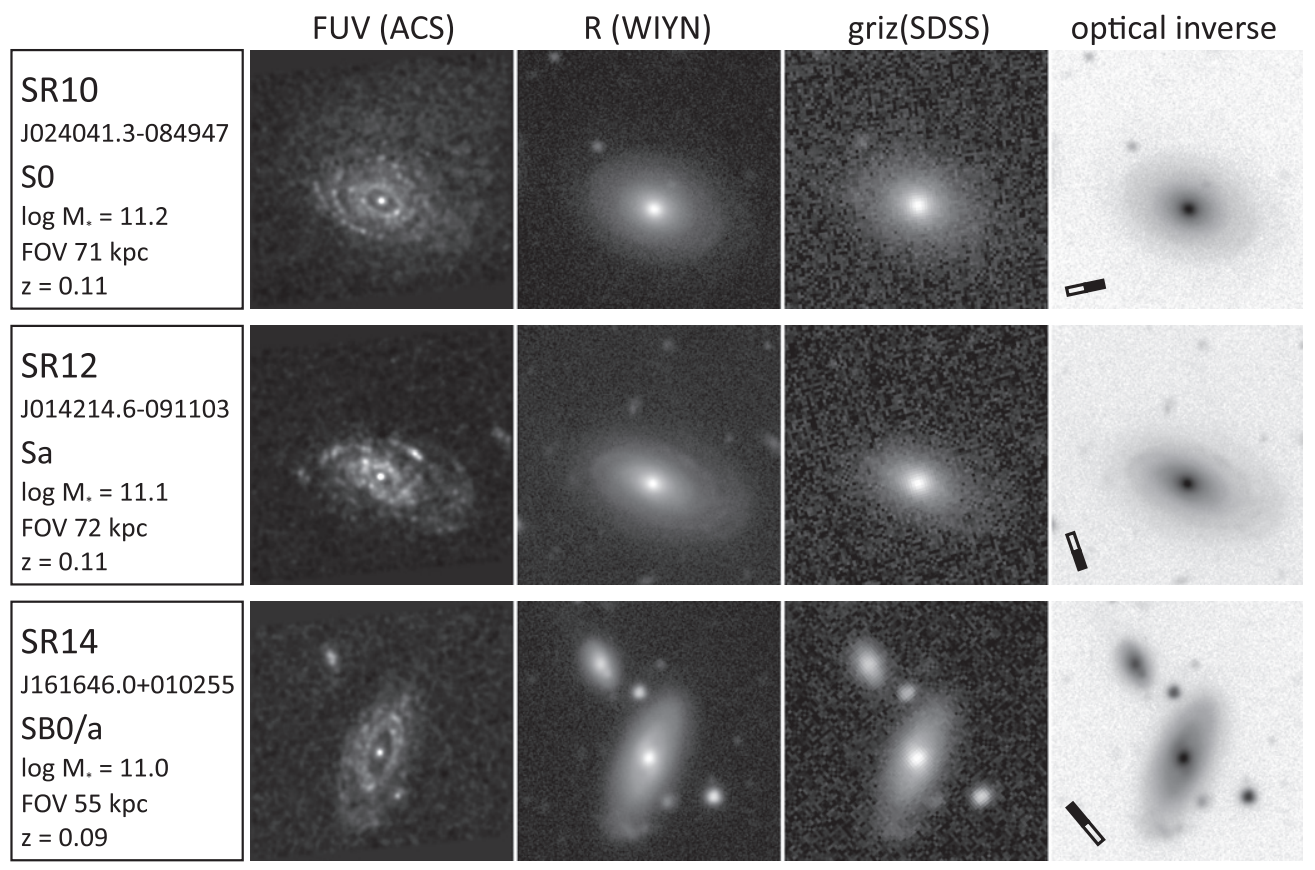

Figure 6. Far-UV and optical images of early-type galaxies with extended SF and having UV disks with small central holes. See Figure 4 for full description. 


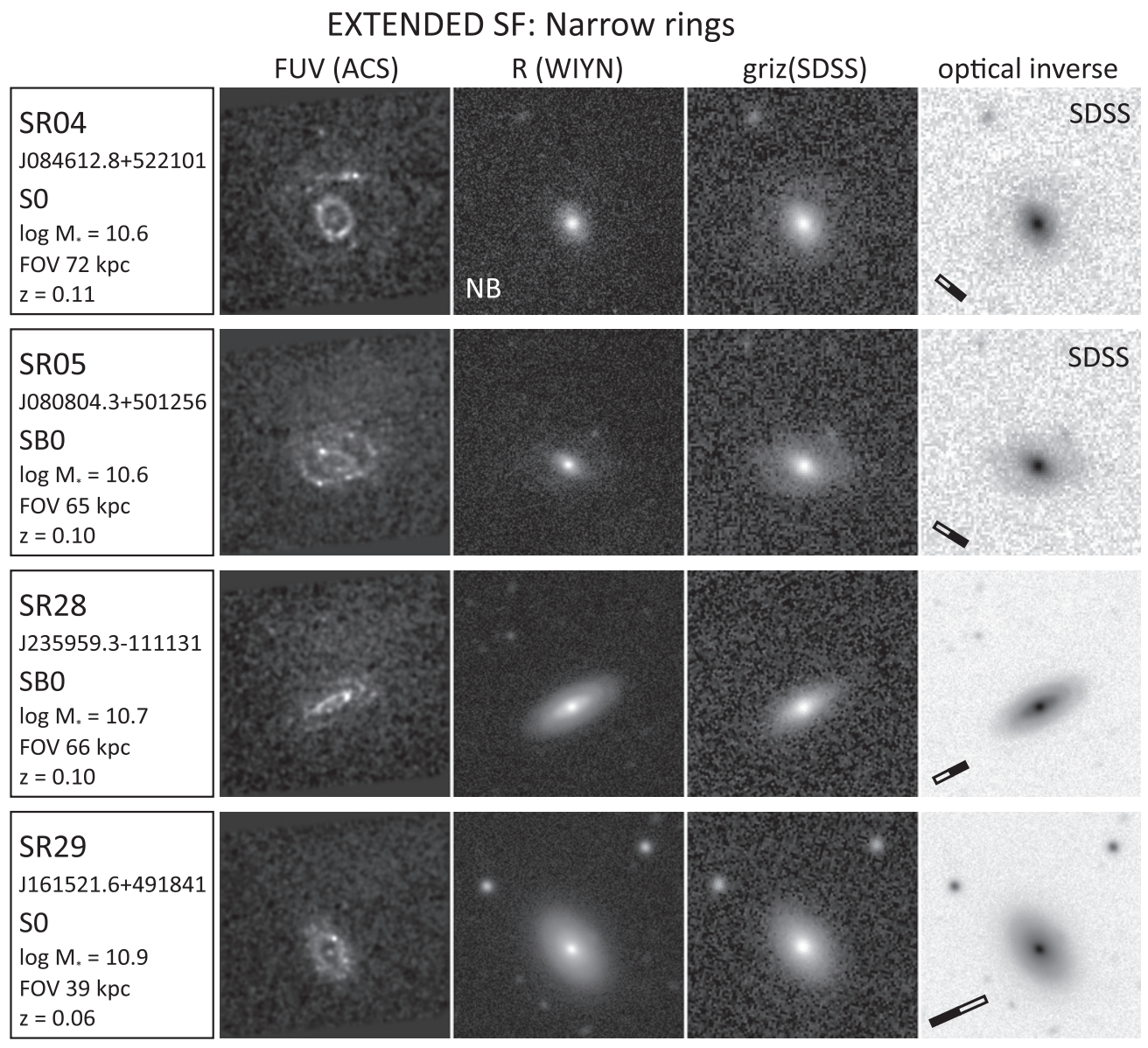

Figure 7. Far-UV and optical images of early-type galaxies with extended SF in the form of narrow UV rings. See Figure 4 for full description. For SR04, the WIYN image does not show broadband $R$ but narrow band within $R$. For SR04 and SR05 the SDSS images are deeper than those from WIYN, so we show them instead of WIYN images in the inverted panels. See Figure 4 for full description.

\section{EXTENDED SF: Irregular rings in extreme disks}

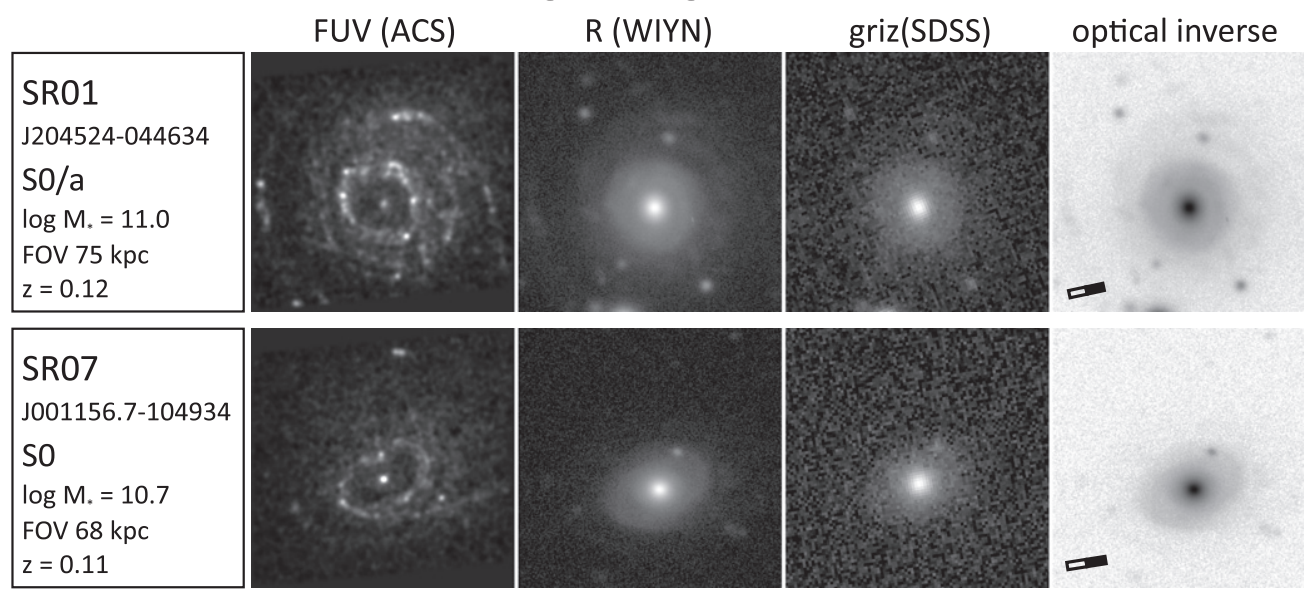

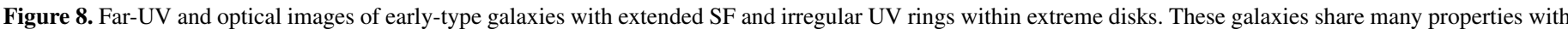
giant low surface brightness galaxies such as Malin 2 and UGC6614 (see Section 10.2.4). See Figure 4 for full description.

images were used to subtract the continuum light present in the narrow band.

Standard observing procedures were followed, however no photometric standards were acquired. Data reduction was performed using tasks in IRAF and L.A.Cosmic (van Dokkum 2001). To facilitate continuum subtraction, we aligned all individual exposures of a single object to the nearest integer pixel. For continuum subtraction of narrowband images, we also convolved all images of a given object to match the PSF of the image with the poorest seeing (typically $0{ }^{\prime \prime} 8$ ). Then the images were co-added in each band with inverse variance as a weight. We determined the scaling of the $R$ image to use for the continuum following Macchetto et al. (1996) by asking that the elliptical isophote at $a=1$.".4, where we know emission lines contribute negligibly, has the same intensity as in the narrowband image. 


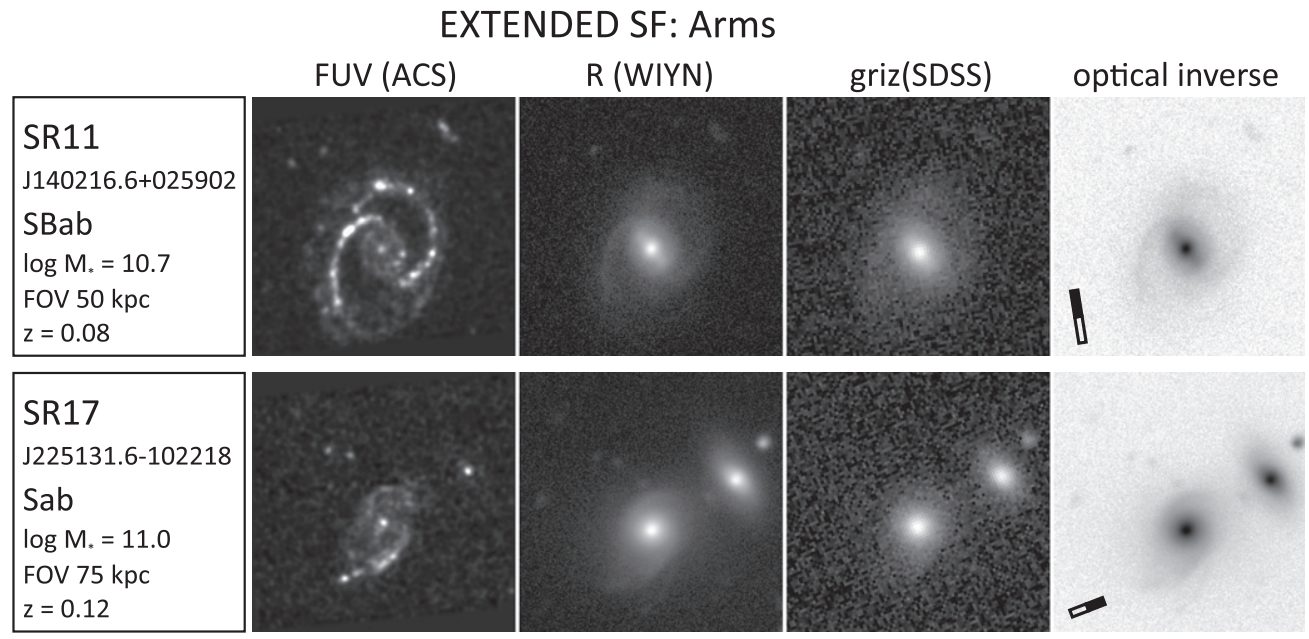

Figure 9. Far-UV and optical images of early-type galaxies with extended SF and UV arms. In SR11 the arms form a pseudoring. Arms in SR17 appear to be tidal, formed by interaction with a companion. See Figure 4 for full description.

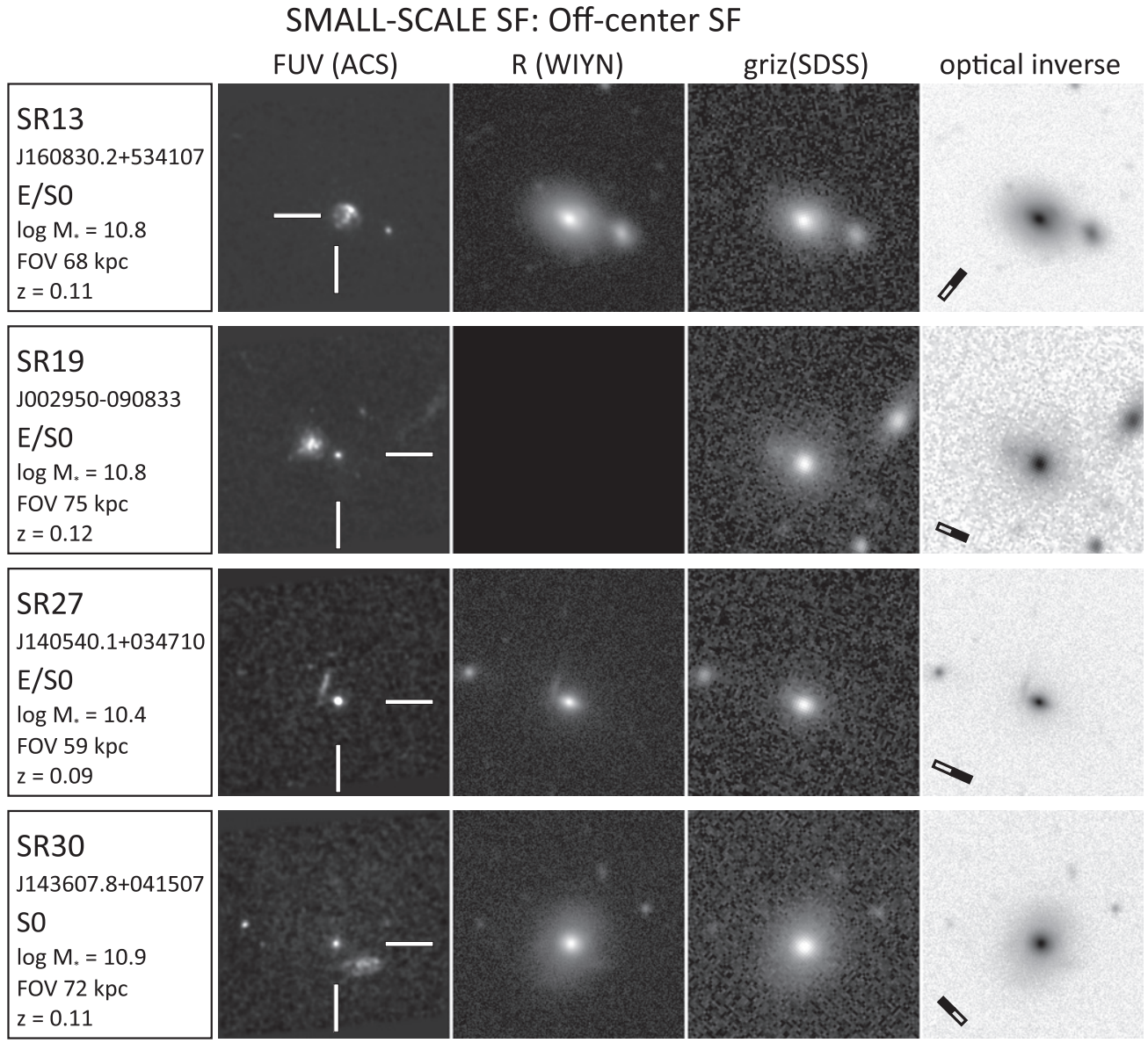

Figure 10. Far-UV and optical images of early-type galaxies with small-scale SF offset from the galaxy center. Crosshairs in the FUV images indicate the location of the optical center of the main, early-type galaxy. See Figure 4 for full description.

To produce the $R$-band images with optimal depth and resolution, which we show in the second column of imaging panels in Figures $4-10$, we co-added images without any PSF degradation, but weighted by inverse PSF FWHM. By comparing the image of one galaxy that is in common to our sample and deep SDSS Stripe 82, we conclude that the resulting depth is approximately 0.5 mag deeper than that of Stripe 82 , or $\mu_{R} \sim 26.5 \mathrm{AB}$ mag $\operatorname{arcsec}^{-2}$.
Figures 4-10 show images of the same object from three different sources: $H S T$, WIYN, and SDSS. To align HST and SDSS images we used coordinate system already set in the headers of FITS images. WIYN observations originally had coordinate system zero points that were many arcminutes off from the true value. To correct them we used the imwcs task from the WCSTools package. This task matches the stars in the frame to known catalogs and updates the FITS headers 
SMALL-SCALE SF: Central SF

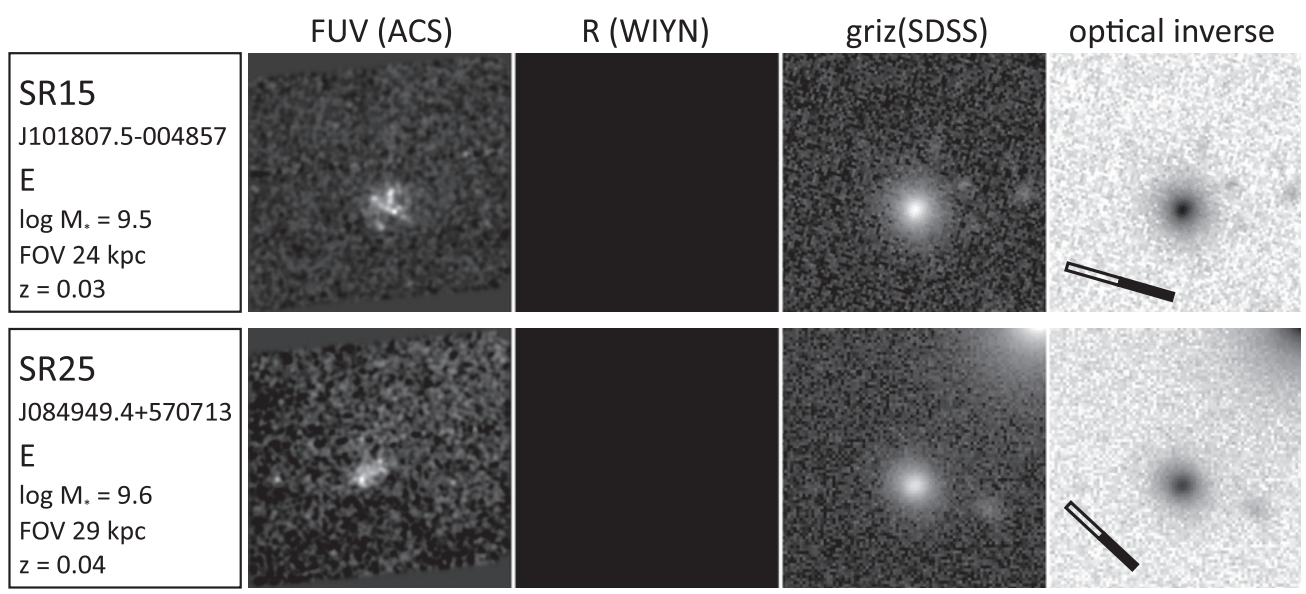

Figure 11. Far-UV and optical images of early-type galaxies with central small-scale SF. See Figure 4 for full description.

\section{UNRESOLVED}

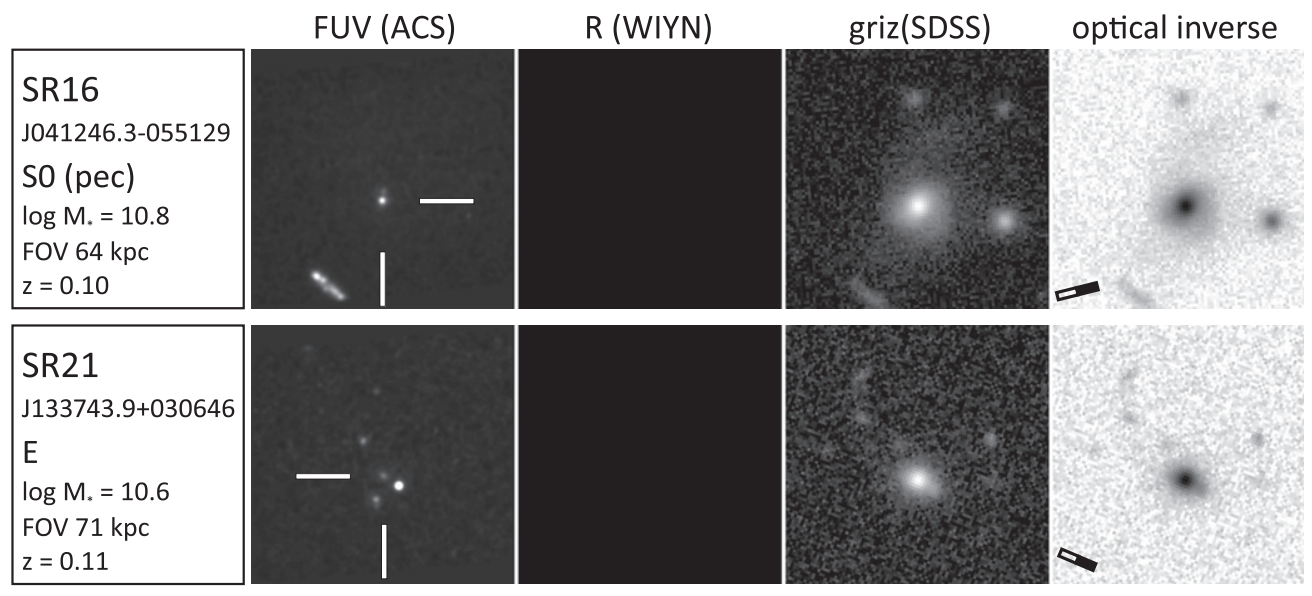

Figure 12. Far-UV and optical images of early-type galaxies with unresolved UV emission. See Figure 4 for full description.

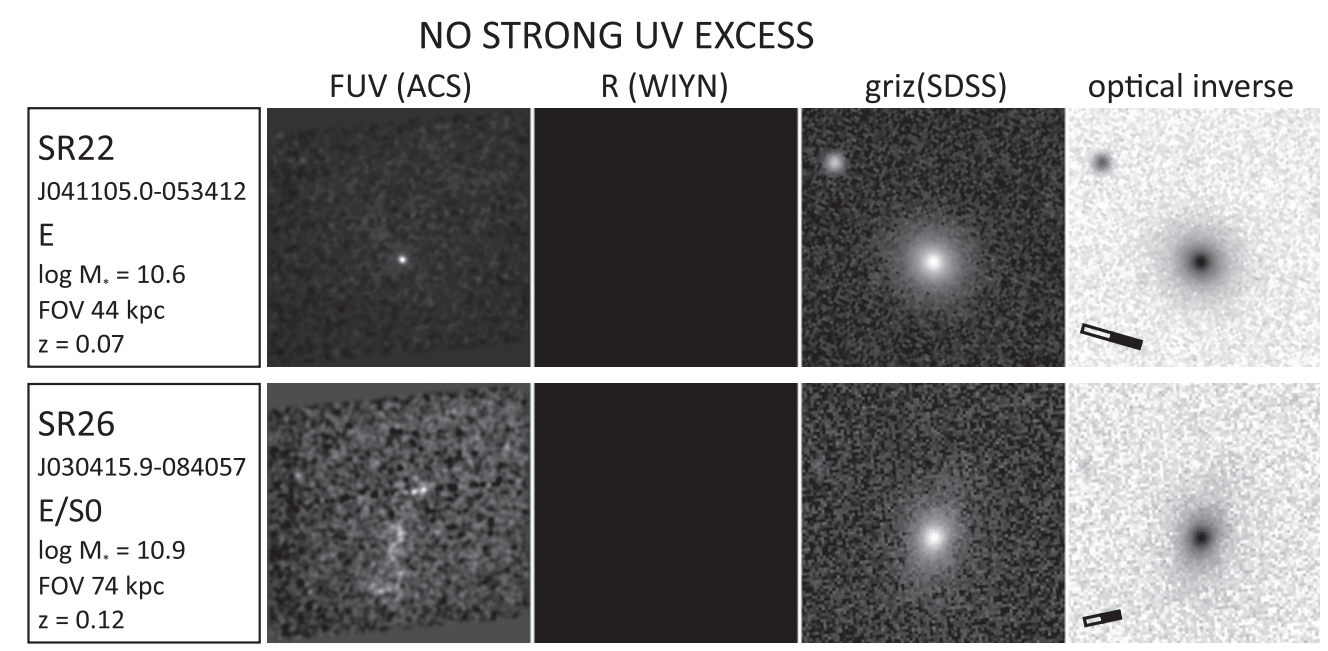

Figure 13. Far-UV and optical images of early-type galaxies with weaker UV excess than the rest of the sample, due to the original FUV magnitudes being underestimated (too bright). See Figure 4 for full description.

accordingly. The catalog to which the matching was done was mostly GSC2. In several cases the task could not obtain an astrometric solution and in those cases the astrometry keywords were modified manually based on measured offsets and known coordinates of the sample galaxy. The accuracy of registration is typically within 1 arcsec.

\section{UV MORPHOLOGY OF THE HST SAMPLE}

We begin the presentation of the results with an overview of UV morphologies of the HST sample of strong-UV excess ETGs. This is a more rigorous and more detailed description and classification than the one provided in SR2010 and is accompanied with the display of all HST UV images. 
As noted in SR2010, the great majority of the sample shows spatially extended and/or patchy structures clearly indicative of star-forming regions. Therefore, the conclusion was reached that for most of the sample the UV excess is attributable to SF. Inferred SFRs are typically $\sim 0.5 M_{\odot} \mathrm{yr}^{-1}$ (Table 1). ${ }^{10}$ The extended/patchy UV emission is not consistent with the emission from old hot populations (the classical UV upturn), which is visible in the HST UV images only as a relatively weak compact or unresolved central source (see discussion in Paper II).

Based on the size of the UV emitting regions, we classify the sample into three main classes.

1. ETGs with galaxy-scale (extended) SF,

2. ETGs with small-scale SF,

3. ETGs with unresolved UV emission.

We place the galaxy into class (1) if the UV emission is present on scales similar to or larger than the apparent optical extent of the galaxy in deep optical images, i.e., if the ratio of the FUV diameter down to $27 \mathrm{AB}$ mag $\operatorname{arcsec}^{-2}$ to the optical diameter corresponding to the $90 \%$ of the Petrosian flux is greater than one. For galaxies in our sample this typically translates into UV diameters greater than $25 \mathrm{kpc}$. We refer to the part of the sample that exhibits galaxy-scale SF as extended star-forming early-type galaxies, or ESF-ETGs. Out of 27 galaxies, $19(70 \%)$ fall in this category, making it the dominant UV type (shown in Figures 4-9).

Galaxies in class (2) show resolved UV-emitting regions that are smaller than the optical extent of the galaxy, i.e., whose FUV to optical size ratio is smaller than $\approx 1$. In our sample they typically have UV diameters smaller than $15 \mathrm{kpc}$, and the UV emission is often offset from the optical center. There are six such cases in our sample $(22 \%)$, and they are shown in Figures 10 and 11 . Finally, two galaxies $(7 \%)$ are classified as UV unresolved, since none of the sources present within the optical extent of the galaxy is resolved (Figure 12). We proceed by discussing the UV morphology of each class and introducing several subclasses. Comparison with optical morphology will be presented separately in Section 7.

\subsection{Extended Star-forming ETGs (ESF-ETGs)}

While star-forming rings are not a very common feature of normal star-forming galaxies (see, for example, the GALEX Atlas of Nearby Galaxies; Gil de Paz et al. 2007), a quick glance at Figures 4-9 reveals that they are the ubiquitous among our ESF-ETGs (only 1 out of 19 galaxies in this category does not possess a UV ring). Despite this common property this class is far from uniform. To facilitate the discussion that will attempt to relate different UV morphologies to their optical properties and possible evolutionary scenarios, we proceed by dividing the 19 ESF-ETGs into five subclasses. As any visual classification the one presented here is to some extent subjective, and may represent categorization of continuous properties, but we believe that it helps systematize complex morphological information. Note that our spectroscopic selection criterion would select against in-filled disks because of their higher central SF. However, as we show in the Appendix, such morphologies are the minority (1/3) compared to those present in the $H S T$ sample, so while they may not be overwhelming,

\footnotetext{
10 All estimates of stellar mass and SFR, as well as the specific SFR, come from Salim et al. (2007) and are determined using Bayesian UV/optical SED fitting and extensive stellar population synthesis models that include dust attenuation. They are given in solar units and for Chabrier IMF.
}

the UV rings will still dominate $(\gtrsim 2 / 3)$ the extended UV morphology in ETGs.

Regular wide rings. We start by focusing on the most populous subclass within the extended star-forming class: ETGs with regular wide rings. Eight galaxies belong in this group, and their UV images are shown in the first column of imaging panels in Figures 4 and 5. All rings are characterized by a relatively uniform, symmetric appearance. This regularity on large scales does not imply a lack of features. For example, SR02 appears to have flocculent structure in the ring, but unlike typical flocculent spiral galaxies (e.g., NGC 4414) this structure is restricted to the ring, and is not present in the inner region. SR06 shows several bright compact star-forming regions within its ring. None of these features, however, takes away from the overall regularity and axial symmetry. A possible exception is the ring of SR18, which appears incomplete, but is perhaps just much fainter on one side. SR08 has a UV bright, compact companion whose FUV flux is blended with that of the main galaxy in GALEX photometry. However, the UV flux of the companion is actually two times smaller than that of the extended emission of the main galaxy, so this ETGs remains a genuine strong UV-excess source (with intrinsic FUV $-r=5.0$ ) even after the companion flux is accounted for. The UV ring is lopsided, a possible result of non-destructive interaction. Altogether, regular rings (with possible exception of SR18) suggest that the SF is not a result of violent processes such as galaxy interactions or mergers. Rather, the distribution of the starforming gas, whether it is internal or external in origin, is related to more quiescent processes including the secular effects of a bar that can lead to ring morphology (Schwarz 1984). Rings in this subclass typically extend to $32 \pm 4 \mathrm{kpc},{ }^{11}$ and have surface brightness in FUV between 26 and $28 \mathrm{AB}$ mag $\operatorname{arcsec}^{-2}$ (Paper II).

Disks with small central holes. We classify three galaxies as belonging to the subclass of ESF-ETGs exhibiting disks with small central holes or clearings (Figure 6). This category is similar to the one we just discussed. A distinction is being made because the central clearing is much smaller than the extent of the ring, which may point toward a different origin. Also, the overall sizes are larger $(41 \pm 6 \mathrm{kpc})$. We also find that the mean SFR of this subclass is significantly higher than that of regular wide rings $\left(1.3 M_{\odot} \mathrm{yr}^{-1}\right.$ versus $\left.0.4 M_{\odot} \mathrm{yr}^{-1}\right)$. Certainly, there could be borderline cases between these two categories, such as SR06 of the regular wide-ring type. Another distinct feature with respect to regular wide rings is that these disks are less uniform on smaller scales than in the previous subclass. One can discern multiple concentric or filamentary arm-like structures. Such features may indicate that the UV structures, despite many similarities with wide rings, are closer in origin to normal spiral disks. We will return to this point in Section 6.

Narrow rings. Four ESF-ETGs are classified as featuring narrow rings (Figure 7). The main characteristic of this subclass is that the rings are both relatively narrow (1-2 kpc) and small $(13 \pm 2 \mathrm{kpc})$. There is a greater diversity within this subclass than in the previous two. Three of the four galaxies in this group (SR04, 05, 28) show signs of an additional, outer ring. In SR04, the bright streak above the middle of the frame may represent the bright part of a very faint outer ring. Its narrow ring is relatively uniform. In SR05 the outer ring is more complete but

\footnotetext{
11 "Errors" of average size indicate the standard deviation of sizes. Sizes represent diameters and were estimated visually. They are accurate to several $\mathrm{kpc}$, depending on how well defined the edge of the UV light distribution is.
} 
is somewhat offset from the inner ring, a possible aftermath of an interaction. Both the inner and the outer rings of SR05 have bright features in them. SR28 has an incomplete outer ring, which may also be a part of a (former) spiral arm. UV structure in SR28 is difficult to determine due to the high inclination. The inner ring appears more complete but is not uniform. Only SR29 shows a single, relatively regular ring (there is only one bright region in it). What makes it belong to this category rather than the regular wide ring class is its rather small size $(14 \mathrm{kpc})$ and narrowness. Non-uniformities seen in this group may indicate that the UV structures were shaped by processes that involved interactions.

Irregular rings in extreme disks. Only two galaxies are classified as belonging to this remarkable subclass of ESFETGs: SR01 and SR07 (Figure 8). Their UV structures are seen to span an enormous extent of 75 and $68 \mathrm{kpc}$, respectively. Both have irregular inner rings of roughly the same size, surrounded by filamentary structures in the very extended disk. Both the misshapen inner ring and the extended UV structures point toward a different origin of SF gas than in any of the previous categories.

ESF-ETGs with arms. The remaining two ESF-ETGs are placed in the category exhibiting well-defined arms, although the two are quite different in appearance and are each unique in our sample (Figure 9). SR11 presents one of the most stunning UV morphologies in our sample, which is very different from what one expects to see in a red sequence ETG. The most prominent features are the two arms that emanate from an inner ring. The arms almost appear to connect in an outer ring. This phenomenon has been described in optical images of nearby galaxies as a "pseudoring" (Buta \& Crocker 1991), and we will discuss the mechanism for its formation when describing the optical morphology of this galaxy (Section 6). The second armed galaxy, SR17, shows less regular arms. Indeed, one arm is more prominent than the other, with different extent and pitch angle. Optical evidence (Section 6) will show that this galaxy is interacting with its neighbor and that these arms are probably tidally induced. SR17 is also the only ESF-ETG for which there is no evidence of a UV ring. This absence and the strong evidence for interaction may be related.

To summarize, the great majority of ESF-ETGs show relatively undisturbed and regular UV morphology, but notable exceptions should not be overlooked. Also, the presence of UV rings in all but one case is intriguing. While our selection criteria would favor such structures, they are likely intrinsically the most common type of extended UV morphology in ETGs (see the Appendix).

\subsection{Small-scale Star-forming ETGs}

Six galaxies in our sample have resolved UV emission that is significantly smaller than the optical extent of the galaxy. We subdivide them into a group of four in which the UV emission is offset from the galaxy center, and two where it is distributed centrally. The latter two galaxies are also very different in terms of their much lower stellar mass than the rest of the sample, which further justifies this separation.

Off-center small-scale SF. We show the four galaxies classified in this group in Figure 10. While there are general similarities in all four off-central cases (such as the size of the star-forming patch of $\sim 10 \mathrm{kpc}$ ), there are some interesting differences. SR13 has a highly asymmetric star-forming patch that encompasses the optical center of the galaxy but is brightest
$5 \mathrm{kpc}$ in projected separation from the center. Optical images show an optical enhancement coincident with the brightest UV region. There is also a separate unresolved UV source corresponding to a likely companion galaxy further out (some $15 \mathrm{kpc}$ away). Next, in SR19, the star-forming patch, located some $9 \mathrm{kpc}$ from the center in projection, again shows multiple bright UV clumps. An optical counterpart is faintly visible too. The center of the main galaxy is an unresolved UV source that appears relatively bright in the UV but nevertheless contains only $10 \%$ of the total FUV flux. In SR27 the extended UV feature shows much less structure and is quite narrow. One-half of the UV emission comes from this feature; the rest comes from an unresolved UV source at the center. Finally, in SR30, the star-forming patch (at a projected distance of $9 \mathrm{kpc}$ ) shows somewhat non-uniform, elongated structure and accounts for $80 \%$ of the UV flux. It is faintly visible in the optical image as well. The star-forming patches in SR19, SR27, and SR30 could be gas-rich dwarfs being disrupted and absorbed by the ETG, but not yet having reached the center like SR13. After accounting for the UV flux in off-center companions, the central unresolved emission in SR19, SR27, and SR30 does not have a strong UV excess in FUV $-r$ and is consistent with the old-star UV upturn.

Since the UV emission in this class is not centered on the optical emission of the main optical galaxy, we need to ask if the off-center structures represent physically associated components or companions, or merely background or foreground "contaminating" galaxies that in GALEX low-resolution images were blended with the main galaxy. In SR27 and SR30 the UV morphology of the companions is relatively undisturbed, so these are candidate interlopers. In order to estimate how likely such a scenario is, we evaluate the incidence of blending in $G A L E X$ images using the following procedure. The FUV magnitude of the main galaxies in this class (without the offset, potentially unrelated, UV flux) is $24.1 \mathrm{mag}$ at the faintest. Using the FUV galaxy counts from $\mathrm{Xu}$ et al. (2005), we "generate" galaxies down to FUV $=24.4$, the faintest an ETG without SF would be at these redshifts, and distribute them randomly over $10 \mathrm{deg}^{2}$. The area of the mock catalog is arbitrary, but it should be large enough to provide reliable statistics. Then we go through this artificial catalog and identify pairs (or groups) that are separated by $<5^{\prime \prime}$, which corresponds to the maximum separation of the off-center UV "companions." Such pairs/groups would be blended in GALEX photometry which is derived from 5" FWHM images. We merge these pairs/groups and recompute the FUV magnitude of the blend, noting the number of components that comprise the blend. Out of 34,700 sources in the original catalog, 361 original pairs and one triple become blended. Based on this simulation of blending we determine the blending probability as the fraction of sources with the integrated magnitude similar to that of the sample $(20.5<$ FUV $<22.5)$ that were originally more than one source. We find this probability to be $3.1 \%$. Consequently, in our HST sample of 30 galaxies we expect one companion to be an interloper. Note, however, that this one interloper will preferentially be found among the sources in which the UV flux of the main galaxy itself is faint, such as those in the class of small-scale star formers. Therefore, it is quite conceivable that either SR27 or SR30 is a chance superposition of an ETG and an unrelated UV-bright galaxy. Future observations could provide a definitive answer, but the main conclusion drawn in this section that gas-rich satellites are consumed by ETGs remains a likely explanation for most of the objects in this class. 
Central small-scale SF. Most of our sample galaxies are relatively massive ETGs with stellar masses in the range $10.2<\log M_{*}<11.2$. Two exceptions are SR15 and SR25 with much lower masses of $\log M_{*}=9.5$ and $\log M_{*}=9.6$, respectively (Figure 1). These are also among the faintest UV sources in our sample. HST UV imaging of them is shown in Figure 11, and they both feature central small-scale starforming regions $2.3 \mathrm{kpc}$ across. Due to the high level of noise, it is difficult to say more about the structure of these regions; SR15 possibly shows several UV bright knots. These too may be minor merger remnants, which can more easily boost the UV-optical color of a low-mass main galaxy. The central UV emission appears quite diffuse, which is probably why it led to no detectable emission in SDSS spectra.

To summarize, most small-scale structures in ETGs are nonuniform and possibly related to minor mergers that do not lead to substantial disruption of the main galaxy. Note that the relative dominance of off-center structures with respect to central ones (four to two) does not necessarily reflect the situation in the overall ETG population because strong central SF is selected against in our sample.

\subsection{Unresolved UV Sources and ETGs without Strong UV Excess}

Unresolved UV sources. There are two galaxies in the HST sample in which we do not detect resolved structures indicative of SF. They are shown in Figure 12. SR16 is dominated by a bright unresolved central source. The color profile measured in Paper II shows it to be bluer (FUV $-r \approx 5.0$ ) than any other center in the entire sample, and therefore least consistent with an old population UV upturn. An AGN does not appear a likely explanation given that the UV color is not very blue. Nevertheless, an unusually strong classical UV upturn is probably the least problematic explanation, since any unresolved nuclear SF would be detectable in the SDSS spectrum. The other galaxy in this class, SR21, shows four unresolved sources within the optical extent of the galaxy, of which the brightest is offset from the optical center of the main galaxy and could be a compact burst of SF in a companion galaxy. As measured in Paper II, the fainter central source itself is too red to be SF, so is probably from UV upturn as well.

Most galaxies classified as extended SF ETGs and smallscale SF ETGs contain a compact UV source in addition to the more extended emission. Despite their sometimes high UV surface brightness these unresolved central sources contain only a small fraction of the total UV flux. The nature of this emission is discussed in more detail in Paper II. The FUV $-r$ colors of the central regions are consistent with emission from oldpopulation UV upturn. Apparently such stellar emission appears rather compact even in 0 '! 3 resolution FUV imaging.

ETGs with no strong UV excess. Finally, we briefly discuss two galaxies which entered the sample due to the erroneous original GALEX photometry. These are shown in Figure 13. SR22 has unresolved UV morphology and, based on the latest GALEX catalog (GR6) and confirmed by measurements in Paper II, has FUV $-r \approx 6.7$, consistent with a weak old-star UV upturn. SR26 also no longer qualifies as having a strong UV excess - its FUV flux is actually very faint. It tentatively shows filamentary extended structure and is thus possibly SF related but appears quite unlike anything in our "normal" extended SF sample. The sources in this category will not be analyzed in the rest of the paper.

\section{IONIZED GAS EMISSION}

The absence of detectable $\mathrm{H} \alpha$ emission in SDSS spectra of the sample appears to contradict the SF seen in the UV. SR2010 discussed two possibilities that would resolve this problem. One is that no $\mathrm{H} \alpha$ emission is present in the central $3^{\prime \prime}$ region sampled by SDSS fibers but that such emission exists further out. The fiber diameter is one-tenth of the size of panels in Figures 4-13. This explanation is certainly consistent with the ring morphologies that dominate most of the sample (see also the Appendix). SR2010 provides an estimate that if one assumes that the measured UV flux is due to SF, the expected $\mathrm{S} / \mathrm{N}$ in $\mathrm{H} \alpha$ would be some 10 times higher than the observed limits. To this argument we add one caveat. Such estimate did not take into account the fact that the strong red continuum present in ETG bulges would act as a high background and drive the $\mathrm{S} / \mathrm{N}$ of the $\mathrm{H} \alpha$ flux down. Therefore, if we now consider the $\mathrm{H} \alpha$ equivalent width (i.e., flux divided by the continuum) instead of the flux alone, we find that the sample is still deficient in $\mathrm{H} \alpha$ with respect to what the UV implies, but by a more moderate factor of $\sim 3$. Another proposed explanation was that $\mathrm{H} \alpha$ is genuinely absent throughout the galaxy, either because there is no "instantaneous" SF $\left(\sim 10^{7} \mathrm{yr}\right)$, while the UV would still trace the "recent" SF $\left(\sim 10^{8} \mathrm{yr}\right)$, or because the environment is not conducive to highmass SF (Krumholz \& McKee 2008). The absence of $\mathrm{H} \alpha$ due to stochastic SF is not expected because the SFRs are several orders of magnitude higher than the expected onset of this phenomenon (e.g., Lee et al. 2009). To test these scenarios and establish if instantaneous SF is detectable at large scales, we conducted narrowband $\mathrm{H} \alpha+[\mathrm{N}$ II] imaging as described in Section 3.3.

Narrowband imaging was attempted for 12 galaxies in the sample: 10 belonging to the extended SF class (SR01, 02, 05, $06,08,11,12,14,17$, and 29) and two in the small-scale SF class (SR27 and 30). Out of 10 ESF-ETGs, we confidently detect $\mathrm{H} \alpha$ emission in three galaxies (SR01, 12, 14), and tentatively in another three (SR02, 11, 17). Neither of the small-scale star formers yielded a detection. Two of the detections are shown in Figure 14. For SR14 (UV disk with a central hole), H $\alpha$ appears as a ring whose extent roughly follows the brighter, inner portion of the UV disk. Another detection shown in Figure 14 is of SR01, classified as an irregular UV ring within extreme disk. Again, the $\mathrm{H} \alpha$ and the UV morphologies are similar. Some compact $\mathrm{H} \alpha$ regions have no UV counterpart and may not be associated with the galaxy.

On average the three galaxies with confident $\mathrm{H} \alpha$ detections are brighter in the FUV than those with tentative detections, which in turn are on average brighter than those with no detections. Therefore, $\mathrm{H} \alpha$ detection appears primarily related to sensitivity and not the intrinsic presence of ionized gas. The absence of $\mathrm{H} \alpha$ in the SDSS spectra serves as a reminder that the information from fiber spectra cannot always be extrapolated to entire galaxies.

\section{OPTICAL MORPHOLOGY OF THE HST SAMPLE}

One of the primary reasons for obtaining new optical imaging in this work was to be able to determine a more secure optical classification than what is possible with SDSS images alone. At the typical redshift of our sample $(z \approx 0.1)$ distinguishing between Hubble types can be challenging. Especially difficult is the distinction between S0s (lenticulars) and pure ellipticals (Es) (Cheng et al. 2011). This separation is often ambiguous even in nearby, well-studied galaxies (Kormendy et al. 2009). 

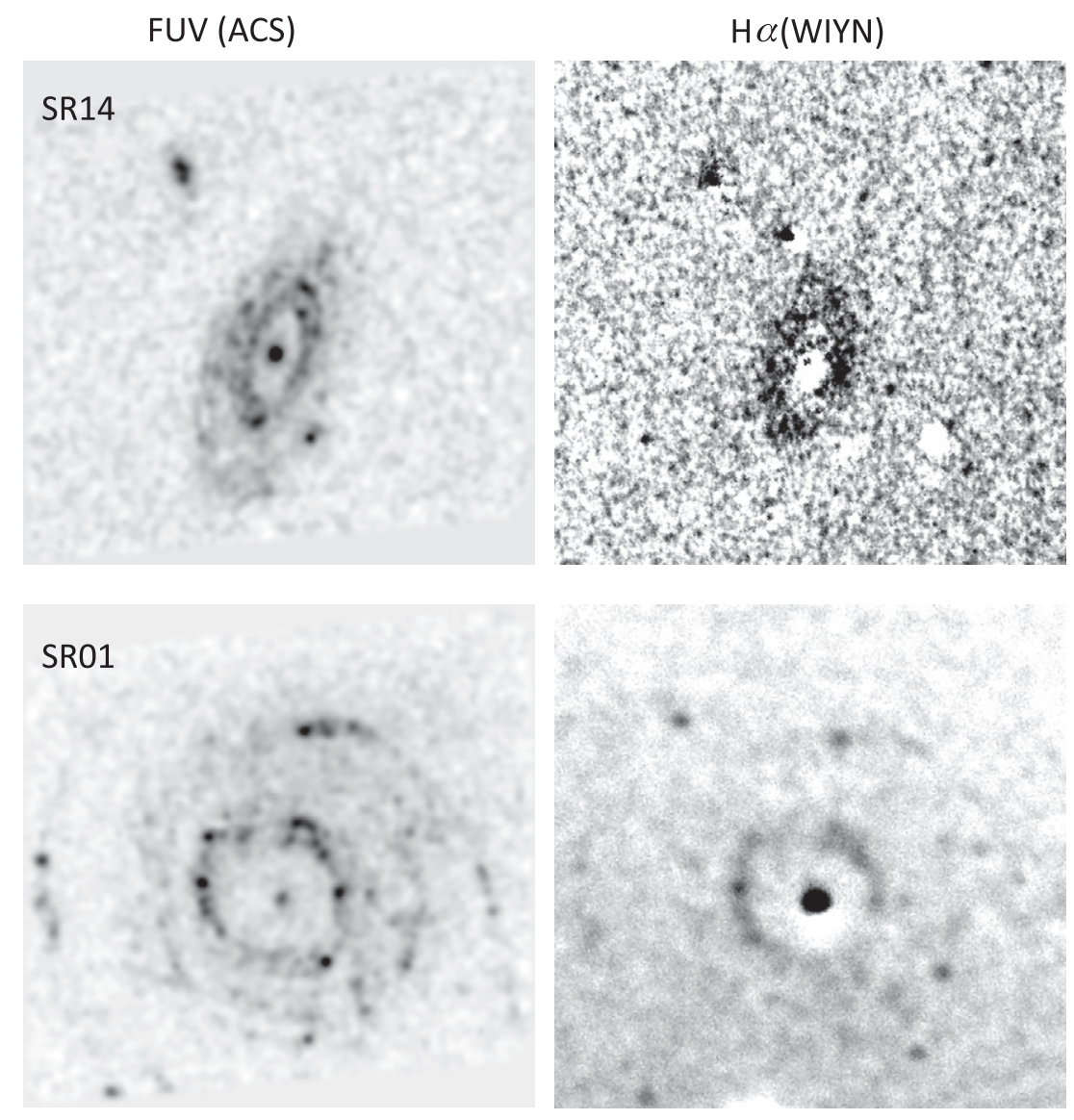

Figure 14. Two early-type galaxies with prominent $\mathrm{H} \alpha$ emission. On the left is the HST ACS far-UV image, and on the right is the continuum-subtracted H $\alpha+[\mathrm{N}$ II] narrowband image. One other galaxy (not shown) has confident $\mathrm{H} \alpha$ detection out of 10 ESF-ETGs imaged. H $\alpha$ is more likely to be detected in galaxies with brighter UV emission, indicating that the ionized gas emission is probably present in all UV emitters, but only detected in more luminous cases.

Deeper and sharper WIYN optical images are available for 18 out of the 27 galaxies in the HST sample ${ }^{12}$ and are shown in the second column of panels in Figures 4-10. Considering that not all galaxies have new imaging, we approached the classification in the following way. We started by classifying based on the SDSS optical image ( $\mathrm{griz}$ co-add) alone (third column of panels in Figures 4-10), assigning preliminary Hubble types and noting uncertainties. We used the criteria of Cheng et al. (2011) to distinguish between Es and S0s. The prime criteria were elongation due to a moderately edge-on disk and, if the galaxy was face on, the sharpness of the edge in the outer profile. A sharp fall-off was taken to represent the edge of an exponential disk, and a gradual fall-off was taken to represent the slower decline in an outer de Vaucouleurs or high-Sersic profile. Then, the classification of 18 galaxies based on WIYN imaging was performed independently. This classification was generally less ambiguous. Comparing the new classification to the preliminary one, we noticed that the new types tend to be later; e.g., many galaxies that looked as if they could be either S0s or Es from SDSS were now classified as S0s. In addition to this, bars were revealed in several cases in which they were not visible in SDSS images. Trying to correct for this "low-resolution" bias, we revisited the SDSS classification of galaxies having no WIYN imaging and assigned final Hubble types. In several cases we still could not decide between E and S0 type and left their type as "E/S0" classification. Hubble types are given in Figures 4-10 as well as Table 1.

$\overline{12}$ The two sources without strong UV excess are excluded from consideration.
Overall, the sample is dominated by $\mathrm{S} 0$ galaxies (17, of which 15 are S0 and 2 are S0/a). Four are later than S0 (two Sa and two Sab), and six are earlier (three E/S0 and three E). Since the original SR2010 selection visually screened the candidates and removed obvious late-type interlopers, the relative lack of galaxies later than $\mathrm{S} 0$ is expected. Even the four later-type galaxies are not typical $\mathrm{Sa}$ or $\mathrm{Sb}$ spirals. Rather, they look like S0s with certain spiral-like features. More surprising is the dearth of true ellipticals (spheroids lacking disks) compared to S0s. We will discuss this absence in more detail later (Section 9).

An especially important optical morphological feature in the context of this study is the presence of a stellar bar. Our sample is dominated by star-forming rings and the classical mechanism for forming and maintaining such rings is the secular dynamical effect of a bar (Buta \& Combes 1996). We find evidence for bars in eight galaxies. This represents $30 \%$ of the full sample, or $38 \%$ of galaxies with secure disks (i.e., excluding E/S0s and pure Es). This rate is roughly consistent with the $30 \%$ observed in more nearby S0s (Aguerri et al. 2009) but falls quite short of the fraction of the sample exhibiting UV rings (86\% of galaxies with secure disks). Apparently, bars are not the dominant mechanism for maintaining rings in our sample.

Optical morphological disturbances are another feature that could provide a clue to the origin of star-forming gas in our sample. Schawinski et al. (2010) and Kaviraj (2010) show that the disturbed optical morphologies in ETGs, such as shells, tidal debris, or dust lanes, are correlated with a UV excess, thus connecting recent SF to mergers. Is the opposite true as well, i.e., are ETGs with a UV excess, such as our 
sample, necessarily morphologically disturbed? To reach their conclusions, Schawinski et al. (2010) and Kaviraj (2010) relied on deep SDSS imaging of Stripe 82, which is 2 mag deeper than the regular SDSS imaging. Our WIYN imaging offers similar, or slightly higher, increase in depth over the nominal SDSS, so it should be sensitive to features detected in these studies. However, we see no evidence for shells or tidal debris in any of the 18 galaxies for which there is WIYN $R$-band imaging. One galaxy (SR17) appears to have tidally induced tails, and there is a nearby companion that shows optical disturbance as well. Only one galaxy shows a dust lane (lower left of SR08 disk, classified as regular wide ring; Figure 4), with two more (SR10 and SR14, both disks with small central holes; Figure 6) showing possible hints of dust lanes. However, the latter look like spiral dust lanes, so they are not merger signatures.

Overall, the optical morphologies in our sample are very relaxed. Even in cases in which smaller companions are present, there is no optical evidence of strong disturbance.

\section{RELATION BETWEEN THE OPTICAL AND THE UV MORPHOLOGIES}

In this section, we examine the connection between the optical and UV morphologies of the HST sample. A strong correlation between UV and optical morphologies would suggest that recent SF may be preferentially found in certain types of ETGs. This would provide useful insight into how SF is regulated in ETGs, possibly hinting at a morphological dependence on feedback processes. Also, the relation between optical features such as the bar and the UV rings would help explain the latter. Finally, the analysis of the UV and optical sizes of galaxies may reveal their SF history.

\subsection{UV Classes versus Hubble Types}

We represent the relation between UV and optical morphological types as a schematic table in Figure 15. Hubble types are grouped into three categories: $\mathrm{Sa}$ (including $\mathrm{Sab}$ ), S0 (including $\mathrm{S} 0 / \mathrm{a}$ ), and $\mathrm{E}$ with E/S0 (i.e., uncertain E or S0). The number of galaxies of each optical type and UV class/subclass is given in a respective cell. The fraction of the given optical type with respect to the total number of galaxies of a given UV (sub)class is shown by the shading of the corresponding cell: light shading indicates lower fraction. A striking result is that none of the 19 ETGs with extended SF is classified as E/S0, and the majority $(80 \%)$ are classified as $\mathrm{S} 0 / \mathrm{a}$. This provides a strong indication that the galaxy-scale SF may not be happening in true ellipticals but only in galaxies already containing disks, i.e., S0 and later.

We next discuss each UV morphological class and subclass in relation to the optical types present in it. S0s dominate among regular wide rings ( $88 \%$ ). The only wide ring galaxy that was classified as a later type (SR02, SBa) does not have very prominent arms. Out of the three disks with central holes, SR12 is classified as an Sa and the other two as S0 and SB0/a. SR12 has well-defined arms, while for SR14 (SB0/a) there is some ambiguity as to whether the arms are the brighter portions of the ring faintly visible in the optical. Later types appear somewhat more likely in this UV subclass. S0s are the only optical type in which narrow or irregular rings occur, though the numbers are small to assess the true incidence of later types. Finally, both galaxies belonging to the UV arms subclass appear armed in the optical as well and are therefore classified as later types. However, these arms are much less prominent in the optical than in the UV. In the case of SR17, as already discussed, the arms

\begin{tabular}{|l|r|r|r||r|}
\multicolumn{1}{c}{} & \multicolumn{1}{c}{ Sa } & \multicolumn{1}{c}{ S0 } & \multicolumn{1}{c}{ E } & Total \\
\hline Extended SF & 4 & 15 & 0 & 19 \\
\hline \hline Wide rings & 1 & 7 & 0 & 8 \\
\hline Disks w/holes & 1 & 2 & 0 & 3 \\
\hline Narrow rings & 0 & 4 & 0 & 4 \\
\hline Irregular rings & 0 & 2 & 0 & 2 \\
\hline Arms & 2 & 0 & 0 & 2 \\
\hline
\end{tabular}

\begin{tabular}{|l|r|r|r|r|}
\hline Small-scale SF & $\mathbf{0}$ & $\mathbf{1}$ & $\mathbf{5}$ & $\mathbf{6}$ \\
\hline \hline Off-center & 0 & 1 & 3 & 4 \\
\hline Central & 0 & 0 & 2 & 2 \\
\hline
\end{tabular}

\begin{tabular}{|l|l|l|l||l|}
\hline Compact & 0 & 1 & 1 & 2 \\
\hline
\end{tabular}

Figure 15. Breakdown of Hubble types with respect to UV morphology class and subclass. Shades of gray represent relative frequency in each (sub)class. Barred and non-barred types are combined. Each type includes subtypes: Sa column includes two galaxies typed as $\mathrm{Sab}, \mathrm{S} 0$ includes two $\mathrm{S} 0 /$ a galaxies, and $\mathrm{E}$ includes four $\mathrm{E} / \mathrm{S} 0$ (uncertain $\mathrm{E}$ or S0) cases. There is a clear trend such that galaxies with extended SF favor later Hubble types ( $\mathrm{S} 0$ and later) while galaxies with small-scale SF tend to have earlier Hubble types.

appear to be tidal in origin, and both itself and the bright optical companion show signs of disturbance. In the other galaxy with UV arms (SR11) they almost close to form a pseudoring. In the optical this closing is much less evident.

The situation is quite the opposite for galaxies classified as having small-scale SF. Five out of six are classified as E/SO or $\mathrm{E}$; only one is a clear S0. From this albeit small statistic, we can speculate that in the same way in which the extended SF in ETGs appears to favor S0s, so could more compact and more central SF favor ellipticals. We will return to this question in Section 10.3.

For the unresolved UV sources, one (SR16) is a peculiar S0 and the other (SR21) is an E. Given that the source of UV in SR16 is not clear (possibly old stars), we cannot place much weight on the fact that this galaxy, unlike most others in the sample, does show some evidence for disrupted morphology. For the other galaxy, the bright unresolved UV source can be traced to the faint optical "companion."

\subsection{UV Classes versus the Presence of a Bar}

We continue our analysis of the relation between UV and optical morphologies by focusing on the incidence of stellar bars in galaxies of various UV classes. Bars are the primary mechanism believed to form stellar rings, so their presence in our sample dominated by UV rings would be of great importance. We represent this information in a schematic table in Figure 16.

We find no bar in any of the six small-scale star-forming ETGs or in the two ETGs with unresolved UV sources. Given that most of these galaxies are classified as E/SOs or Es, no bar is expected. The presence or absence of bars is of much more significance for the 19 ESF-ETGs, of which 18 have rings, which, if of non-collisional origin, are believed to require bars to produce and perhaps also to maintain them. 


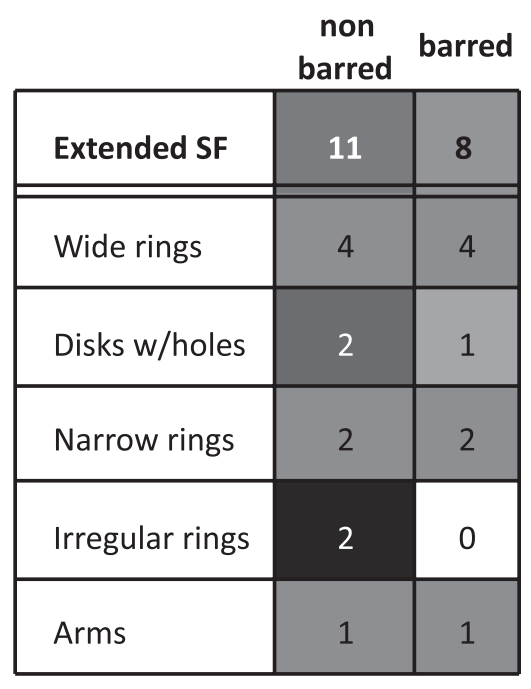

\begin{tabular}{|l|c|c|}
\hline Small-scale SF & 6 & 0 \\
\hline \hline Off-center & 4 & 0 \\
\hline Central & 2 & 0 \\
\hline
\end{tabular}

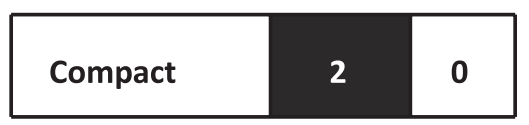

Figure 16. Breakdown of barred vs. non-barred disks with respect to UV morphology class and subclass. Shades of gray represent relative frequency in each (sub)class.

Out of eight galaxies exhibiting regular wide rings, whose smooth morphology makes them prime candidates for resonance rings, one-half shows evidence for a bar and the other half does not. The presence or absence of a bar, while notable in the optical, does not reflect itself in any obvious difference in the UV. The UV rings have the same extent regardless of the presence of a bar, and they appear to be equally well defined. Of the galaxies with regular wide rings, one stands out in terms of its optical morphology. SR03 is the only galaxy in our sample that has a conspicuous optical ring that is detached from the bulge of the galaxy. The ring appears to be held in place by a very strong bar, one of only two such strong bars in our sample. While there are several optical detached rings that have been studied in local S0s (e.g., NGC 1291 and NGC 1543, both barred), neither their optical nor UV morphologies fully match that of SR03. Namely, in SR03 the bar extends all the way to the ring, making it presumably a corotation ring, while in locally studied galaxies the corotation ring is basically an inner ring, imbedded in a disk or surrounded by a larger outer ring (Buta \& Combes 1996; Buta 2011). An optical ring means that, unlike other galaxies in our sample where the ring is mostly a feature composed of young stars, here the older stars are co-located in the ring, indicating that it has been present for most of the lifetime of the galaxy, or that the dynamical processes were able to migrate the stellar population alongside the star-forming gas.

Moving to other UV classes, only one of the three disks with small central holes appears to have a bar (SR14). Galaxies with narrow rings are evenly split between barred and non-barred, with no significant differences in UV morphology. Neither of the galaxies classified as irregular rings in extreme disks has a bar. Given the irregularity of the rings, it is quite possible they are not of the resonant type, which means that bars are not expected to play any part in their formation or maintenance.

Of the two galaxies with UV arms, SR17 has no ring and no optical bar. On the other hand, SR11, with arms forming a pseudoring, has a strong bar. This bar has an associated inner ring, thus representing a classical resonance corotation ring. A good local example of a galaxy with very similar UV and optical morphologies is UGC12646 (Buta \& Combes 1996). Altogether SR11, with its bar, inner ring, and outer pseudoring, is very well explained by bar-induced dynamical processes, convincingly reproduced in numerical simulations (Schwarz 1984; Athanassoula et al. 2009)

To summarize, we find that among the ESF-ETGs the barred and non-barred galaxies appear quite similar in the UV. The range of their UV-based SFRs (determined using SED fitting, see Salim et al. 2007) largely overlap, although barred galaxies on average have $\log$ SFR $=-0.34 \pm 0.06$, while non-barred have $\log \mathrm{SFR}=-0.16 \pm 0.07$, a somewhat significant difference $(2 \sigma)$, but in the opposite direction from what might be expected. Therefore, the presence of a bar probably does not lead to higher SFRs in our sample.

\section{IMPLICATIONS OF UV AND OPTICAL SIZES FOR STAR FORMATION HISTORY}

In this section, we will deduce some aspects of the SF history of the galaxies in our sample by analyzing their UV and optical sizes. First, we try to establish whether the current SF is part of a long-term process that leads to disk buildup in ETGs, and then we explore whether the relation between the optical and UV sizes can point toward the origin of the gas responsible for SF.

\subsection{Current SF and the Optical Disk Re-growth}

What is the relation between the current SF and the optical disk? If we assume that the optical disk requires prolonged SF to build up, the question can be asked whether the current episode of SF visible in the UV is related to a renewed, additional disk building after the galaxy got onto the optical red sequence (i.e., are stars being made beyond the "original" extent of the disk), or whether it is the fading last phase of the original disk SF, confined within the "expected," non-enhanced extent.

To try to answer this question we compare the optical sizes of our HST sample galaxies to an underlying population of quiescent disk-like ETGs. If the optical sizes are larger than those of the comparison sample, it would indicate that (1) additional disk building took place after the galaxy arrived on the optical red sequence (i.e., after the initial intense SF was finished). If, on the other hand, the optical sizes of ESF-ETGs are comparable to those of quiescent ETGs, it would indicate either (2) that the current episode of SF has not lasted sufficiently long, or has been intense enough to have produced much of the optical disk, or (3) that the SF that we see is just the fading remnant of the SF that produced the original, normal-sized disk. Case 1 would arise from processes such as ETG rejuvenation through a steady, long-lasting intergalactic medium (IGM) accretion; case 2 would result from either very low level accretion or from a transient minor-merger-induced SF, while case 3 would be consistent with a recent arrival onto the optical red sequence, i.e., the final phases of SF quenching. For this test we use optical, $r$-band sizes to minimize the "artificial" boosting of sizes of ESF-ETGs at shorter wavelengths due to ongoing SF. In other 


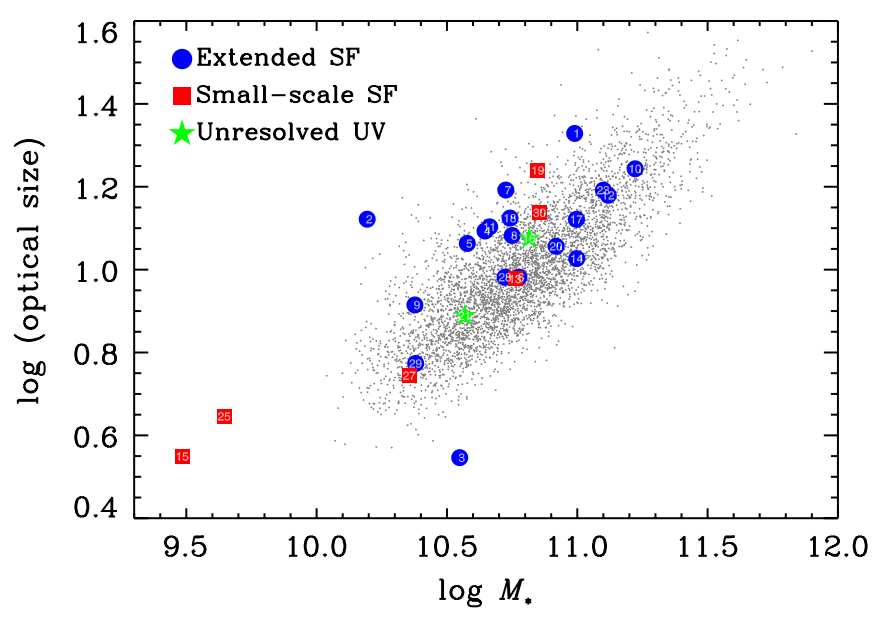

Figure 17. Comparison of optical disk physical sizes of the HST sample (various colored symbols) and the underlying population of quiescent disk-like ETGs, at a given stellar mass. Size is measured as the radius containing $90 \%$ of the Petrosian flux in $r$ and is given in kpc. The HST sample is split into UV morphology classes: extended SF (blue dots), small-scale SF (red squares), and unresolved (green stars). Numbers inside symbols indicate object identifications (SR\#). The underlying sample is generally assumed not to have experienced additional disk growth after getting on the optical red sequence, allowing a comparison with the HST star-forming sample to show any subsequent buildup. SR01, 02, and 07 are most significantly larger than the underlying population.

(A color version of this figure is available in the online journal.)

words, the assumption is that fading alone (case 3) will not affect the optical size measured in the $r$ band.

To characterize the optical disk sizes we use physical sizes corresponding to radii encompassing $90 \%$ of the Petrosian flux in $r$ band (rest-frame $V$ ). We verify that this choice of radius is a good measure of the full extent of disks by overlaying them on optical images. To select the comparison sample of quiescent disk-like ETGs, we choose those with the similar redshift range as the sample ESF-ETGs $(0.08<z<0.12)$, with red UV-to-optical and optical colors to ensure quiescence (FUV $-r>6$ [including UV non-detections] and $g-r>$ 0.7 ), and with optical light concentrations like that of the sample $(2.5<C<3.2)$. The upper cut is the maximum concentration of the HST sample and is meant to reduce the contamination from pure spheroids. Note that any remaining spheroid contamination will be adding galaxies with sizes larger than disky ETGs, since at a given mass we find that ETGs with $C>3.2$ are larger than those in the disky range of $C$. The assumption is that most of the quiescent disk-like ETGs did not experience additional disk building upon arrival on the optical red sequence and can thus serve to show reference sizes that result from the original disk building. The results are shown in Figure 17, where we plot the physical sizes of the HST sample and the comparison population against stellar mass. Blue circles represent ESF-ETGs, red squares are small-scale star formers, and green stars are unresolved UV galaxies. Numbers within the symbols provide galaxy IDs. Overall, ESF-ETGs tend to be larger than the corresponding red-sequence ETGs, though they range from significantly larger to similar in size, and even a few undersized. ${ }^{13}$ Formally, we find using a K-S test that the distribution of the residuals of optical sizes of ESF-ETGs with

\footnotetext{
13 SR03, the galaxy with the detached optical ring, is the only galaxy in the HST sample significantly below the trend. We verify that in this case the SDSS radius encompassed only the bulge/bar region and none of the disk/ring, therefore severely underestimating the optical extent of the galaxy. The actual size is some 3.6 times greater, making this one of the galaxies that is larger than what is typical for its mass.
}

respect to a nominal size-mass relation defined by quiescent ETGs has a probability of only $2.4 \times 10^{-3}$ to have been drawn from the same size distribution as the quiescent ETGs. The galaxies that most stand out are SR01,02 and 07. These include both galaxies that are classified as irregular rings in extreme disks, while the third galaxy (SR02) is classified as a regular wide ring. While the presence of both extreme disks among the "oversized" galaxies does not appear to be a coincidence, there is no clear-cut connection between the UV class and the level of disk size enhancement. Of the five ESF-ETGs that are visually identified as having some SF outside of where we can trace the optical extent (SR01, 02, 04, 06, 07), four have oversized optical disks, which suggests that the current SF will lead to further growth of optical disks.

To summarize, we see evidence that a part of the sample $(\sim 1 / 2)$ experienced some level of disk building ( $\sim 50 \%$ increase in size) with respect to other disk-like ETGs, and the SF that we see is very likely related to that process (see also arguments in Paper II). This new disk-building phase apparently proceeds in the same plane as the existing disk, at its outskirts. The other half has disk sizes that suggest that the currently observed SF has not played a major role in disk building after most of the original SF ceased, either because disk enhancement involving external gas has started more recently, or because the SF is using up the original internal supply of gas.

\subsection{Comparison of UV and Optical Disk Sizes}

While most of the UV extent in ESF-ETGs is found within the optical extent of the galaxy, this does not mean that the UV light profile follows that of optical light. As shown in the analysis of the surface brightness profiles in Paper II, the optical profiles drop more steeply outward than the FUV profiles, which are mostly flat. In other words, the FUV to optical colors become bluer away from the center by some $2-4 \mathrm{mag}$. We can address the connection between the UV and optical, and therefore between younger and older populations, using a morphological analysis as well. Specifically, systematic differences in the ratio of UV to optical disk sizes can help us distinguish between the fading of the original SF and the possible subsequent episodes of SF, which would preferentially happen at the outskirts of a galaxy. In the previous section, where we compared the optical sizes of star-forming and quiescent disks, we were only able to identify likely cases of significant buildup due to SF subsequent to the arrival on the red sequence, while the small-level buildup (including accretion that started/resumed more recently) and the disk fading would not have led to the increase in optical sizes. Therefore, the analysis of the ratios of UV to optical disk sizes can provide further information on the processes that lead to SF.

The next test consists of comparing the UV to optical size ratio of the HST sample of ESF-ETGs to the size ratio of other massive galaxies with similar, intermediate specific SFRs (i.e., the dust-corrected green valley population). The assumption now is that most green valley galaxies are fading for the first time onto the red sequence (Martin et al. 2007). Thus, the size ratios similar to theirs would support the fading star-forming scenario for the HST sample (Section 10). Therefore, we select the comparison sample to have the same mass range as the ESF-ETGs $\left(10.2<\log M_{*}<11.2\right)$ and lie in the similar redshift range $(0.08<z<0.12)$. For the FUV size we take the FWHM measurement from the GALEX pipeline, and for 

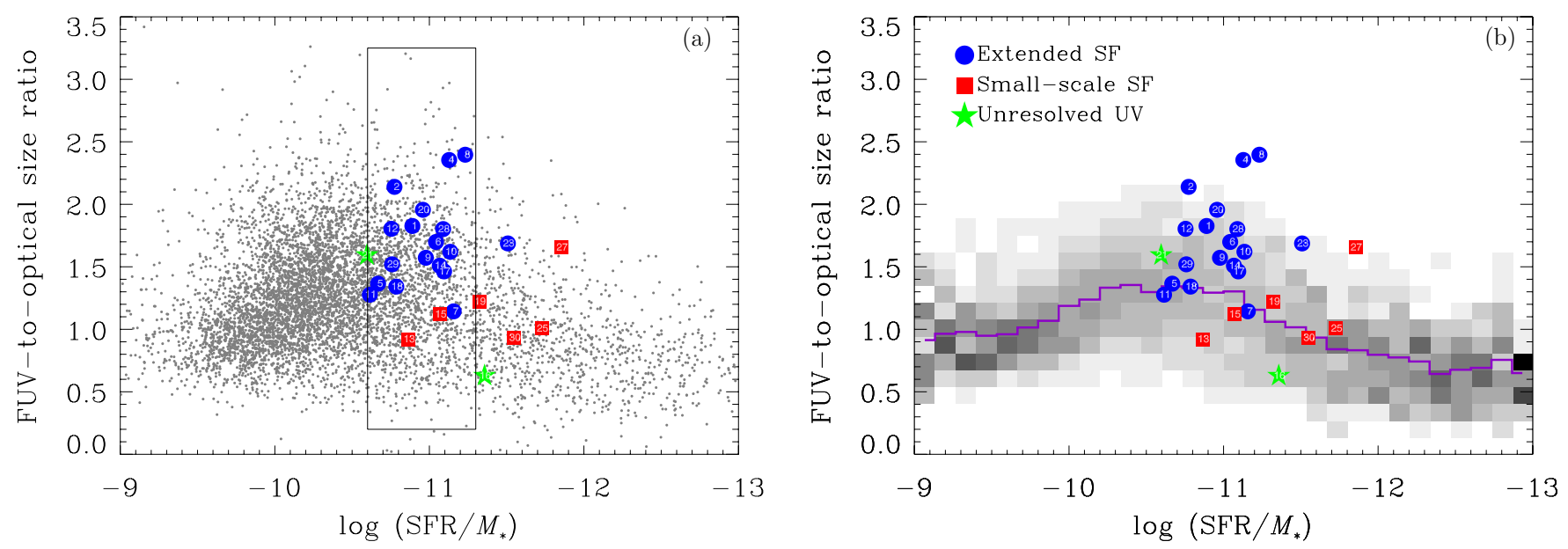

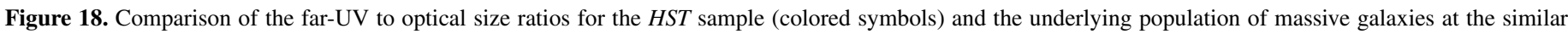

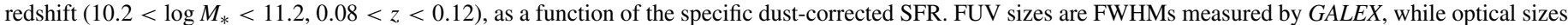

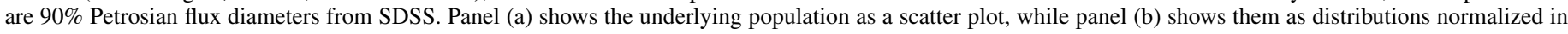

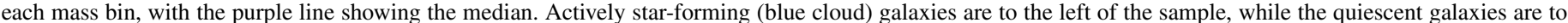

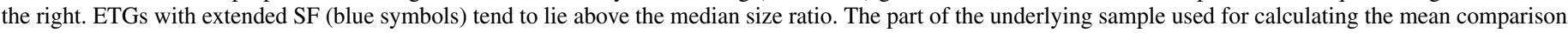

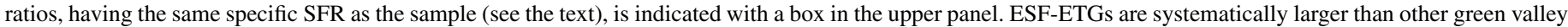
(intermediate specific SFR) galaxies of similar mass.

(A color version of this figure is available in the online journal.)

the optical size the $90 \%$ Petrosian flux diameter in $r .{ }^{14}$ The results are shown in Figure 18(a), where the size ratio is plotted against the dust-corrected specific SFR. Blue cloud star-forming galaxies are to the left of our sample, while the quiescent ones are to the right. Our sample spans intermediate specific SFRs, as expected from the FUV $-r$ excess selection. Small-scale star-forming ETGs (red squares) all tend to have smaller UV to optical size ratios than ESF-ETGs, and also have smaller specific SFRs. Unfortunately, at these redshifts GALEX FUV size measurements are quite uncertain for any individual galaxy, so commenting on size ratios of individual galaxies may not be very revealing. Instead, we check if on average the ESFETGs differ in size ratio with respect to other galaxies of same specific SFR. To perform the comparison, we focus on the specific SFR range of $-11.3<\log \left(\mathrm{SFR} / M_{*}\right)<-10.6$ and size ratios between 0.1 and 3.3. These cuts leave two ESF-ETGs outside: SR03 with greatly underestimated optical size (leading to unrealistic formal size ratio of 4.9) and SR23, an outlier in terms of low specific SFR. Expanding the cuts to include these objects would bias the size ratio of the underlying sample toward even lower values. We calculate the geometric mean of the ratios of ESF-ETGs to be $1.66 \pm 0.09$. The corresponding value for the comparison sample is $1.25 \pm 0.01$. Monte Carlo simulations of random drawings of 17 galaxies from the underlying sample show that the mean ESF-ETGs size ratio is expected to be larger than that of the underlying population by chance with a probability of $3.8 \times 10^{-4}$, corresponding to a $3.4 \sigma$ difference. Therefore, the UV sizes of ESF-ETGs are on average larger than what is expected for transitioning galaxies with fading SF, suggesting instead that the gas was accreted from an external source. This result becomes easier to visualize when we show the distribution of the underlying population in the conditional form (normalized in each specific SFR bin) in Figure 18(b). Statistically significant difference in size ratios persist at the similar level even if we exclude seven ESF-ETGs for which we

\footnotetext{
${ }_{14}$ Note that because the FUV and optical size are not measured in the same way, a galaxy with a flat color profile need not necessarily have a size ratio of exactly 1.
}

have already established significant disk enhancement from the analysis in the previous section.

The analysis performed in this section is similar to that presented in SR2010 using FUV physical sizes alone, but is more robust since it avoids potential biases by comparing relative sizes and by selecting the underlying population with the same mass as the HST sample. The results suggest that ESF-ETGs, while having the same dust-corrected specific SFR as the more general population of green valley galaxies, are different from them because their UV emission is distributed more broadly than their optical emission, pointing to a different fueling mechanism.

\section{INCIDENCE OF SF IN S0s VERSUS ELLIPTICALS}

The selection criteria for our sample of ETGs were not explicitly biased in favor of S0s or against true ellipticals, yet we see that none of the ESF-ETGs is an elliptical. A question therefore arises whether the galaxy-wide, extended SF is an exclusive S0 phenomenon. To answer this we go beyond our sample to investigate the incidence of the occurrence of SF among the general population of SDSS/GALEX ETGs.

Since the classification of SDSS galaxies into Es versus S0s is challenging and not readily available in this redshift range, in this section we resort to the fact that Es and S0s do not have the same distributions of stellar mass, optical concentration, or axis ratio. We then determine what fraction of ETGs with a given stellar mass, optical light concentration, or axis ratio have extended SF, and whether these trends indicate if the extended $\mathrm{SF}$ is primarily an S0 phenomenon or not.

We define the underlying sample of ETGs by requiring high optical light concentration $(C>2.5)$, red optical colors $(g-r>0.7$, rest frame), and $z<0.12$, the same redshift cut as the HST sample. All our HST ESF-ETGs are on the optical red sequence $(g-r>0.7$; Figure 1 of SR2010), which basically means that any SF must be at a low level (in the sense of low specific SFR, not necessarily low total SFR). As in Section 8.1 the underlying sample is not required to have a UV detection, only to lie in the GALEX footprint. 
To select candidate ESF-ETGs among the ETGs defined above, we impose two additional requirements: FUV $-r<6$, to select SF, and the UV to optical size ratio $>1.15$ to select extended SF. The FUV $-r$ cut is not as blue as the one used to select the HST sample, where we required a strong UV excess of FUV $-r<5.3$. Studies of nearby galaxies have shown that the old-star UV upturn is not bluer than FUV $-r=6$ (Donas et al. 2007), so it is justified to use it as a cut to select SF. The UV to optical size cut is simply chosen based on Figure 18, such that all ESF-ETGs from the HST sample lie above it. Note that no selection is based on the SDSS fiber properties.

Figure 19 shows the incidence of ETGs (at the given parameter value) that fulfill the extended SF criteria as a function of stellar mass, concentration, and axis ratio. In the upper panel, we see that the incidence of extended SF among the ETGs peaks at $15 \%$ for masses between $10.6<\log M_{*}<11.1$. The range of masses of the HST sample (blue bar) encompasses this peak. Above the mass corresponding to the peak incidence the incidence relatively quickly declines and reaches zero around $\log M_{*} \approx 11.6$, i.e., at masses which are only three times higher than the masses at which SF was still at the peak. While the sample contains ETGs with masses of up to $\log M_{*}=11.9$, their numbers are too small to constrain the SF incidence, i.e., it is consistent with zero. Interestingly, the decline of the SF incidence starts at the same place at which ellipticals take over SOs in terms of number densities (i.e., where the two stellar mass functions cross; Cheng et al. 2011; Vulcani et al. 2011). This is consistent with the extended SF being primarily an S0 phenomenon. The drop at lower masses, where S0s dominate, must be of different origin. One possibility is that since toward the lower masses the fraction of galaxies that are satellites increases (Mandelbaum et al. 2006), and since the satellites usually cannot maintain SF as they fall into a central halo (e.g., Bekki 2009) the incidence of ETGs with extended SF will decrease. This hypothesis will be tested in future work that will consider the environments of ESF-ETGs.

Unlike the trend with mass, the incidence of extended SF among ETGs falls monotonically with increasing concentration, again consistent with a picture in which mainly S0s support extended SF. For the least concentrated ETGs the rate is $\approx 20 \%$. Finally, the SF incidence versus minor to major axis ratio also declines as the galaxies get rounder in projection, but unlike the trend with concentration it levels off as galaxies get round. This again is consistent with SF in S0s, since some fraction of them will be seen face on. The fact that the highest SF fraction $(\sim 25 \%)$ of any of the trends is reached at lowest axis ratios, where true Es are absent $(b / a<0.45$; Cheng et al. 2011), again argues for the connection with S0s.

Bolstered by these results, we attempt to derive an overall incidence rate of extended SF among the "pure" S0s and Es. To select samples with as little overlap as possible between S0s and Es, we take Es to be ETGs that are more massive $\left(\log M_{*}>10.9\right)$, more concentrated $(C>2.9)$, and rounder $(b / a>0.65)$ than the rest of ETGs, i.e., simultaneously using the cuts at which bulge-dominated ETGs start to outnumber disk-like ETGs (Cheng et al. 2011). Applying these cuts greatly decreases completeness, but this is not relevant since we are only interested in the rate of incidence. Analogously, "pure" S0s are selected as simultaneously not fulfilling these three criteria. The results are very indicative. Massive ellipticals selected in this way have extended SF in $3.8 \%$ of cases, while the fraction is $21 \%$ for S0s. At face value, S0s are almost six times as likely to exhibit extended SF. However, the more intrinsically
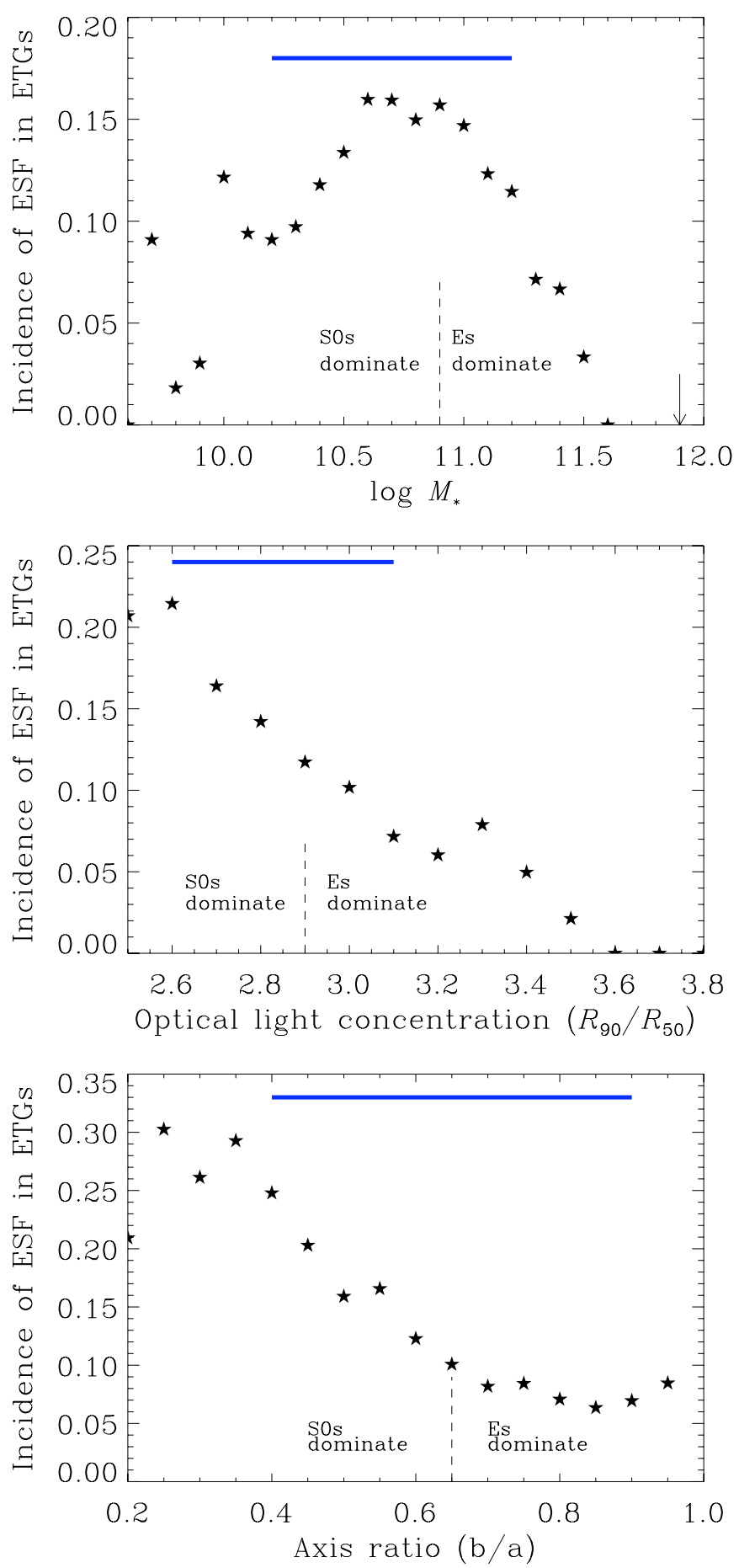

Figure 19. Incidence of extended SF (i.e., SF which results in the UV extent exceeds the optical size) among ETGs as a function of stellar mass, optical light concentration, and optical axis ratio. ETGs are selected as concentrated galaxies on the optical red sequence. The blue bar represents the range of the HST sample. Dashed lines in each panel show values above which ellipticals start to dominate over S0s (according to Cheng et al. 2011; Vulcani et al. 2011). Trends are consistent with extended SF being more frequent in less massive, less concentrated galaxies that can appear more inclined, i.e., in galaxies of S0 type rather than true ellipticals.

(A color version of this figure is available in the online journal.)

rare some objects are in a given group, the larger the relative contamination. In this case, larger relative contamination will be for Es. Since the above cuts produce only 55 "pure" Es with extended SF, one can easily inspect SDSS color images 
of all of them and look for non-elliptical interlopers. We find that a number of galaxies selected as pure Es nevertheless show features incompatible with being an actual elliptical, such as bars and obvious disks. Interestingly, detached optical rings are seen in five objects (there was only one such ring in our HST sample). Altogether, it appears that at least $1 / 2$ of these galaxies are not ellipticals. Thus, the true rate of extended SF in ellipticals is probably smaller than $2 \%$, in stark contrast to the S0 rate. The $21 \%$ incidence rate for S0s is similar to that of extended UV disks (XUVs) of late-type spirals determined by Thilker et al. (2007), possibly suggesting a similar gas supply mechanism(s).

The main focus of this work is on the phenomenon of the extended, galaxy-scale SF. A few cases of more compact SF in the HST sample are probably not typical in the sense that we see them as being mostly off-center, while in reality nuclear and small-scale SF may be as common. To evaluate the incidence of such SF, we use the above methodology but ask that the UV to optical size ratio be smaller than 1.15 . We find that the overall rates of small-scale SF are similar to those of extended SF. Small-scale SF becomes more frequent than the extended SF at the high-mass end $\left(\log M_{*}>11.2\right)$, possibly indicating that ellipticals are more efficient at preventing the extended SF than the more concentrated central SF. Nevertheless, the SF incidence reaches zero at the same high mass as in the case of the extended SF. Other trends are similar to those of the extended SF, with a possible increase of incidence at very high concentrations. We find that the fraction of "pure" Es, with small-scale SF is $7.4 \%$, while it is $13 \%$ for "pure" S0s. This is a smaller disproportionality than in the case of extended SF. However, as in the case of extended SF, the selection will be sensitive to contamination, so the estimates provided here are more likely to be upper limits, especially for ellipticals. Inspecting the SDSS images of "pure" Es with small-scale SF, we again see many that actually appear to have extended SF (optical rings) and there is a large number of non-elliptical interlopers.

\section{DISCUSSION}

The discussion will focus on exploring various mechanisms that can lead to SF in the ETGs on the optical red sequence, and considering which of them may be related with the UV morphologies present in our sample. In general, the galaxies on the red optical sequence are considered "red and dead" because they have not had high global levels of SF in the recent past, yet, as we have seen, some of them are active at a low relative level.

\subsection{Mechanisms Driving SF in ETGs, and the Source of Molecular Gas}

When considering the mechanisms driving SF in red sequence ETGs, it is useful to first define two broad possibilities for the provenance of the star-forming gas: internal and external (e.g., Wardle \& Knapp 1986). Since galaxies in general grow by merging and accretion, it is important to be precise by what is meant here. We consider internal gas to be the material that was present in the disk or the bulge of a galaxy at the time when it (first) arrived on the optical red sequence, i.e., when the intense SF (having high specific SFR) that used to keep it optically blue has ceased. The internal gas would include whatever gas was left over from this original epoch of SF, plus any gas subsequently released by the existing stars (mostly as stellar winds in the evolved stages of stellar evolution). In contrast, all of the gas that entered the optical extent of an ETG after it got on the optical red sequence would be considered external. This includes both the gas acquired from merging with smaller, gas-rich galaxies and the gas accreted from the IGM.

In terms of the mechanisms driving the SF, several scenarios have been proposed in the literature. While they are not all mutually exclusive, they are differentiated here to emphasize the dominant process. We schematically illustrate these mechanisms in Figure 20. Each panel shows the SF history for the given mechanism, as well as the optical classification (upper bar) into blue cloud (BC) and red sequence (RS) and the UV-optical classification into actively star-forming (SF), green valley $(\mathrm{GV})$, and passive. Blue cloud and actively star-forming phases are coincident, while the optical red sequence corresponds to either the green valley (some SF) or the passive phase (no SF). A galaxy is no longer on blue sequence soon after the commencement of quenching. In the discussion here we are generally not interested in what causes the SF to start its decline.

1. Fading of the original SF. In this scenario any SF currently present in red sequence galaxies is simply the final, lowlevel phase of once-intense SF in a disk galaxy. In other words, intense SF has been absent for some time and consequently the galaxy is now optically red, but with enough UV from low-level SF to have "green" UV-optical color. In the UV-optical color-magnitude diagram (CMD), such a galaxy would be considered a transitional galaxy on its way to quiescence (Martin et al. 2007). Note that in the optical CMD this galaxy is already on the red sequence, but would have arrived there recently because some SF is still present (see Figure 20). In this picture the remaining SF would be distributed across the galaxy disk and possibly show fading spiral arms (similar to field "red spirals" of Masters et al. 2010a). Since in this scenario the spiral galaxy does not need to undergo a morphological transformation, the reason it is fading can be assumed to be because of the lack of new IGM accretion (e.g., massive-halo quenching of galaxy groups, Dekel \& Birnboim 2006), possibly coupled with some other non-destructive feedback mechanism that removes the gas. The origin of gas in this model is internal because the galaxy is in the process of exhausting the gas it had prior to arriving onto the optical red sequence.

2. SF due to recycled gas. In this scenario SF is quenched primarily due to the lack of IGM accretion, but the quenching does not proceed all the way to zero. New stars continue to form from the mass loss of evolved stars. In this mechanism the new SF should mostly follow the distribution of old stars, and is consequently more often invoked to explain central SF in ETGs (Temi et al. 2009; Shapiro et al. 2010). Also, unlike scenario (1), one would not necessarily expect to see fading spiral arms because a morphological transformation into an S0 (large bulge and featureless disk) could have preceded the quenching. The main objection to this model is that all ETGs are expected to recycle gas, yet most do not have either nuclear or widespread SF. This is even more of an issue considering that the recycling mechanism should be able to keep supplying the gas to be used in SF for very long periods of time (Kennicutt et al. 1994; Temi et al. 2009; Leitner \& Kravtsov 2011). The origin of gas in this model is internal because SF does not require external input to run its course.

3. Gas-rich minor mergers. Minor mergers, being more frequent than major mergers (Maller et al. 2006), may represent an important way in which galaxies grow and change in general (Bournaud et al. 2007). This process is possibly as prevalent among the ETGs as among the 
(1) Fading of original SF

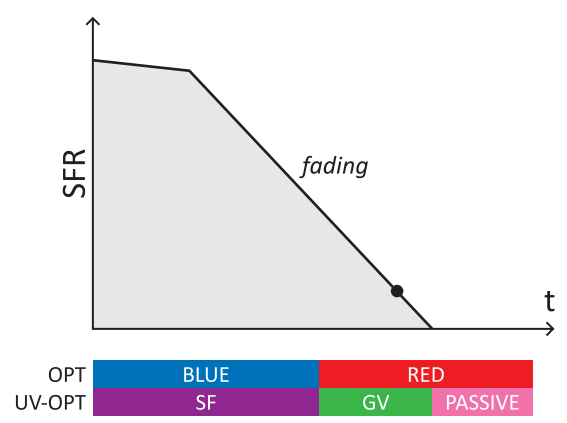

(2) SF due to recycling

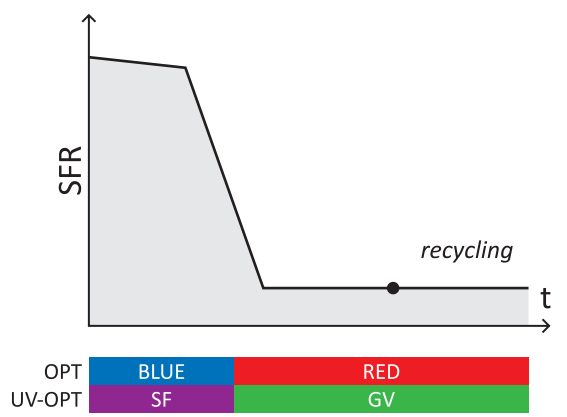

(3) Minor mergers

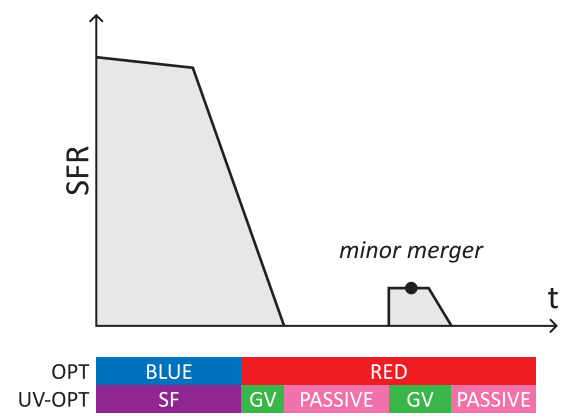

(4a) IGM accretion (intermittent)

(4b) IGM accretion (quasi-static)
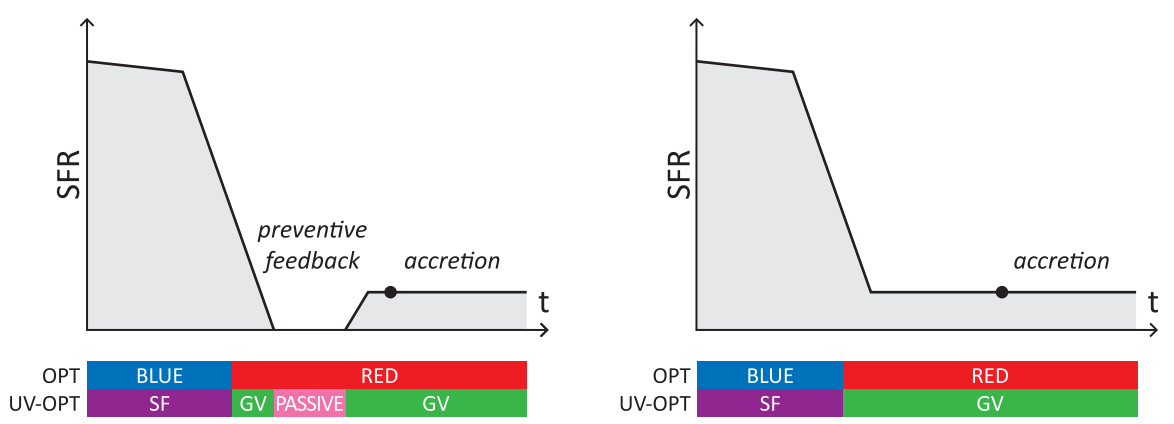

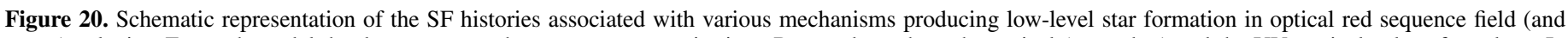

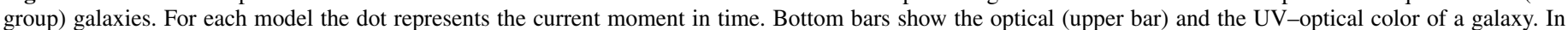

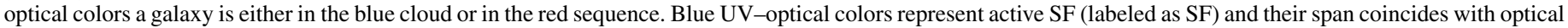

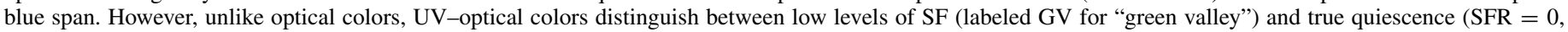
labeled as PASSIVE). A galaxy leaves the blue cloud soon after the quenching starts.

(A color version of this figure is available in the online journal.)

general galaxy population. Therefore, gas-rich minor mergers (typically defined as mass ratio of 4:1 or higher) are difficult to dispute as a mechanism that is taking place among ETGs, which may subsequently lead to episodic SF in ETGs (Figure 20). The key question is how dominant minor merging is as the SF mechanism among ETGs. Kaviraj et al. (2009) claim that principally the entire population of ETGs having strong UV excess can be explained in this way. The success of such explanations to a large extent depends on knowing the timescale over which minor mergers lead to enhanced SF. A factor of a few overestimate in timescale can turn this mechanism from dominant to just one of the contributing mechanisms. One of the main goals of Paper II is to explore episodic SF from the standpoint of stellar populations. The origin of gas in this scenario is external.

The emphasis on mergers being minor stems from the fact that major mergers would be producing more intense bursts, sufficient to drive the galaxy all the way back into the blue cloud. Optically blue ETGs have been identified in several studies based on SDSS data (Kannappan et al. 2009; Schawinski et al. 2009), and may be precursors to post-starburst E+A galaxies. Because major mergers would probably destroy stellar disks, and we see that disks are present in all of our ESF-ETGs, they do not present a viable mechanism for the current $\mathrm{SF}$, though they may have happened in the past.

4. Intergalactic medium accretion. The IGM accretion scenario assumes that the low-level SF present in some ETGs is fueled by newly accreted external gas, i.e., that the original molecular gas is no longer available. This mechanism is especially interesting in the light of recent work that attempts to establish the importance of the so-called cold accretion in supplying the gas to galaxies in general (Kereš et al. 2005; Davé et al. 2011). Note that we use the term "intergalactic" without implying the precise provenance of the gas (which before it enters the galaxy could be circumgalactic, i.e., it may accumulate beyond the stellar disk before it becomes available for SF), but rather to indicate a relatively smooth, possibly prolonged process that stands in contrast to a bursty satellite accretion. ${ }^{15}$ If, as recent studies indicate, the IGM accretion is the dominant mode by which galaxies acquire gas, then its regulation determines if and when a galaxy leaves the blue cloud and becomes optically red in the first place. This scenario was mostly considered in the context of normal, actively star-forming galaxies (e.g., Kereš \& Hernquist 2009). It has recently received more attention in connection with SF in ETGs (Kauffmann et al. 2007; Donovan et al. 2009; Cortese \& Hughes 2009; Thilker et al. 2010; Oosterloo et al. 2010; SR2010).

We distinguish two flavors of this scenario, differing in SF history: intermittent IGM accretion and quasi-static IGM accretion. Both are illustrated in Figure 20. In the first type, the IGM accretion has resumed after some period of true quiescence. The period of quiescence may be the result of the same process that led to the quenching in the first place. That would be the case if the preventive feedback was temporary or is intermittent. SF proceeds when the feedback

\footnotetext{
15 While both the minor merger accretion and IGM accretion represent accretion, to avoid confusion we will use this term in connection with the latter mechanism alone.
} 
is not active. In the second type, the quenching shuts down most of the SF and removes most of the original gas, but there is no mechanism in place to prevent new, low-level IGM accretion. Note that in such a case the galaxy never reaches true passivity, so it can remain in the green valley for a very long period of time, which is why we call it quasi-static.

Both minor mergers and IGM accretion have been proposed as mechanisms leading to XUVs, relating them to inside-out disk building. The phenomenon was originally described in star-forming spirals (Thilker et al. 2007) but has recently been extended to ETGs (Lemonias et al. 2011; Moffett et al. 2012), and so becomes relevant in the context of this study. Furthermore, both minor mergers and accretion might be associated with external reservoirs of $\mathrm{H}$ I such as those found around some ETGs having ongoing SF (e.g., Donovan et al. 2009; Cortese \& Hughes 2009; Thilker et al. 2010). Thus, the evidence for one mechanism versus another must rely on the additional information beyond the presence of neutral gas, such as the UV and optical morphologies explored here, or the inferred SF histories (Paper II).

In relation to the above processes the term "rejuvenation" is sometimes encountered in the literature (Hawarden et al. 1979; Shapiro et al. 2010; Thilker et al. 2010). If rejuvenation is taken to mean any recent SF that can lower the mean age of the stellar population, all of the processes except fading would fall into this category. On the other hand, if rejuvenation is taken to mean the resumption of SF after it has been completely absent (as in Paper II), only the minor merging and the resumed/intermittent IGM accretion would qualify under this term.

So far, we have discussed SF mechanisms without considering the two types of ETGs (Es and SOs). Fading assumes that SF happens in a disk, and therefore it is only applicable to SOs or later types. Minor mergers could in principle operate on either ellipticals or S0s, but the resulting SF morphologies could be quite different in the two cases. In the case of spheroids, a merger may lead to SF that preferentially happens in the centers (Peirani et al. 2010) or forms morphologically very distinct polar ring galaxies (Combes 2006), while for the disk galaxies significant merger-induced SF may happen in an existing disk as well (Mihos \& Hernquist 1994; Cox et al. 2008). A difference in the way in which SF is induced in Es and S0s may also exist for IGM accretion. In S0s gradual accretion could lead to SF in the existing stellar disk, perhaps in its outskirts. On the other hand, the IGM accretion onto ellipticals may result in a detached rotating ring, as in the case of Hoag's galaxy (Schweizer et al. 1987; Finkelman et al. 2011), without any gas cooling onto the spheroid itself.

Finally, note that the fading SF scenario implicitly assumes that S0s form from spirals via some non-destructive mechanism (e.g., strangulation), while the other three scenarios do not imply any specific mechanism for the original transformation of a progenitor into an S0 galaxy.

\subsection{SF Mechanisms in the HST Sample}

With the above review in hand, we now evaluate the SF mechanisms against the morphological evidence for each UV class described in Section 4. We start by considering ETGs with extended SF (ESF-ETGs).

\subsubsection{Regular Wide Rings}

Galaxies with regular wide rings represent the most numerous single class in our sample, and half of all ESF-ETGs. They have fairly undisturbed morphologies both in the UV and in the optical suggesting no gas-rich mergers with mass ratios smaller than 10:1 in the last 1-2 Gyr (Lotz et al. 2011). These rings are very different from the collisional rings and could instead be secular rings where a non-axisymmetric feature such as a bar leads to the formation of rings (Buta \& Combes 1996). Regular morphology argues strongly against mergers or interactions, which are actually believed to be able to destroy the outer rings (Elmegreen et al. 1992). A possible exception is SR18, which shows what looks like an incomplete UV ring coincident with a compact optical source, and has some extended features along the major axis of the optical disk. The presence of rings is compatible with any of the remaining mechanisms (fading, recycling, IGM accretion). The lack of features in rings and the lack of optical spiral structure, as is basically the case here (7 S0s and $1 \mathrm{Sa}$ ), argue against the fading of the original SF which would presumably preserve more structure in either the optical or the UV. Absence of large-scale structure would then favor either recycling or IGM accretion.

As recycling should be present in all S0s, especially in inner regions, and bars that would facilitate the transport of gas from inner regions to the corotation radius are also common, one would expect most barred SOs to exhibit a star-forming ring. We test this hypothesis by extracting 89 prominently barred $\left(L_{\text {bar }} \geqslant 0.5\right)$ So $(-3 \leqslant T \leqslant-1)$ galaxies from the EFIGI catalog of 4458 nearby galaxies with detailed morphological information (Baillard et al. 2011). From these we randomly select 20 that are observed by GALEX and check their UV images. We find that only two exhibit any signs of extended FUV emission: NGC 4643 has a narrow ring at the corotation radius, and NGC 5052 has a somewhat small offset ring. From this quick analysis we conclude that while recycled gas may be present in the majority of ETGs it alone will rarely lead to SF even when a driving mechanism such as a strong bar is present. Since all S0s must be accumulating internal gas in their disks, evidently there is some sort of ubiquitous "cleaning" mechanism that is rendering this gas inactive. To explain the ESF-ETGs this way would require that the mechanism be failing in most S0s. But why would that happen? There is no difference in the properties of these objects that we can see that points to a difference in inherent cleaning efficiency. We therefore assume that cleaning operates in all galaxies and that the presence of excess gas in the ESF-ETGs must be due to extra gas from other sources, not to a pile-up from internal sources. Moreover, this argument applies to all types of ESF-ETGs, not just those with wide rings, and thus we do not consider possibility (2) (SFR from recycled gas) any further in the rest of the analysis.

What evidence is there that the wide UV rings in the HST sample are the result of IGM accretion? A possible indicator is that in half of these cases a bar is not seen. This makes it unlikely that some other global dynamical process can completely remove the internal gas from the central regions. In contrast, external accretion may not need a bar to settle in a ring, since its natively high angular momentum would cause it to remain in the outskirts. Alternatively, the external gas is prevented from reaching the central regions even with a very weak nonaxisymmetric potential, such as a dissolved bar that would not be visible in our optical images. A feature that may suggest such scenario is that the UV rings in this class are wide and usually extend from the inner regions (presumably a corotation radius) out to the very edges, or in some cases, beyond the optical disk. Further evidence for a mechanism that does not involve a bar is the fact that in well-studied nearby resonance rings in late-type 
spirals, where secular processes shuffle the existing disk gas, the corotation ring is narrow and is oftentimes accompanied by an additional, outer Lindblad resonance ring, which we do not see. In NGC 2974 (type S0/a), the only galaxy from the nearby SAURON sample that has UV rings (also embedded within the stellar disk), Jeong et al. (2007) posit the existence of a bar that leads to ring structures through resonances, although the bar is not visible in this galaxy. Remarkably, NGC 2974 is surrounded by a ring-shaped reservoir of $\mathrm{H}$ I coincident with the UV rings (Weijmans et al. 2008), an arrangement that fits the picture presented here in which a conspicuous bar may not be needed if the source of gas is external.

If IGM accretion is the process that leads to SF in these galaxies, it is probably occurring at a rate or timescale that may lead to some enhancement of the existing disk. Figure 17 shows that most members of this subclass lie above the expected size for their mass, with the most extreme case by far being SR02, with its wide, flocculent ring. Such enhancement in size and the overall regularity of UV structures may point toward a prolonged accretion, as in the quasi-static scenario described above (see also Paper II).

In summary, IGM accretion appears as the most viable mechanism for fueling SF in ESF-ETGs with wide rings. We exclude SF due to recycled gas because it is not seen universally among barred S0s. The morphologies are too regular to imply gas-rich mergers and too unstructured to suggest fading spirals.

\subsubsection{Disks with Small Central Holes}

Turning now to the three ESF-ETGs classified as disks with small central holes, the same arguments hold against the minor merger scenario: non-disturbed UV and optical morphologies. Of the remaining two mechanisms, here the fading of the original SF is perhaps more likely than IGM accretion. Indeed, these three galaxies with UV structures reminiscent of tightly wound arms and relatively small central clearings are the best candidates for the fading scenario in our sample. Two, perhaps even all three, have optical signatures of spiral arms. In SR14 a bar is present and it may be responsible for the central clearing. In the other two cases the clearing is very small, so an equally small bar cannot be fully ruled out from our imaging. GALEX Atlas of Nearby Galaxies (Gil de Paz et al. 2007) contains one galaxy, NGC 2841, a flocculent Sb with a classical bulge, that has a central hole in the UV that is very similar to galaxies in this class, especially SR14. Young \& Scoville (1982) have suggested that the clearing in NGC 2841 could be the result of the gas exhaustion in the region of the bulge. Fisher (2006) finds that SF is often times suppressed in spirals with classical bulges (see also Lackner \& Gunn 2012) but speculates that it is due to an AGN feedback. Classical bulges are believed to be the result of mergers and share many properties with elliptical galaxies including the presence of a supermassive black hole (Drory \& Fisher 2007). Classical bulges could then be associated with holes in the UV without a need for a bar. Interestingly, NGC 2841, like galaxies in our sample, has low SFR for its mass compared to other spirals, with a curious absence of H II regions in its disk (R. M. Crockett et al. 2012, in preparation).

Drory \& Fisher (2007) suggest that all galaxies with classical bulge have had their current disks re-formed after the merger that formed the bulge and presumably destroyed an earlier disk. Some evidence that subsequent disk accretion is taking place may lie in the fact that NGC 2841 exhibits an XUV disk (Thilker et al. 2007). In that case, galaxies with a classical bulge may represent extreme cases of rejuvenation that proceeds at a relatively slow pace since the overall optical color of galaxies with classical bulges is red (Drory \& Fisher 2007; Lackner \& Gunn 2012). Nevertheless, we will consider objects in our sample having small central holes to exhibit SF from the original, disk-building phase that is currently fading onto the red sequence. Such a conclusion is also supported by the fact that the optical disk sizes of these three galaxies do not exceed what is expected for their mass, consistent with this being the original SF episode.

\subsubsection{Narrow Rings}

The next category is ESF-ETGs with narrow rings, which are somewhat less regular than the rings in the previous group. Because of this varying level of regularity, the SF in this group may have heterogeneous causes. SR05, with its offset outer ring, offset optical disk and a faint companion, is a possible merger candidate. SR28 may show evidence of UV arms, so it could be another example of the fading category. Its normal optical size supports this. SR29 also has a normal optical size, but interestingly, we again fail to detect a bar that maintains its ring.

\subsubsection{Irregular Rings in Extreme Disks and Giant LSBs}

The two galaxies that we classify as irregular rings in extreme disks (SR01 and SR07) deserve special attention because they have whopping UV disks ( $D \sim 70 \mathrm{kpc}$ ) and their optical sizes by far exceed what is typical for their mass, which makes them potentially very rare objects. SR01 and SR07 are very similar to nearby giant low surface brightness galaxies (LSBs), such as Malin 1, Malin 2, and UGC6614. ${ }^{16}$ SDSS color composite images of Malin 2 and UGC6614 show dominant bulges. Malin 2 in addition has arcs similar to those seen in the $R$ image of SR01. Some arcs are optically red, while some are blue, indicating more intense SF. UGC6614 shows an inner blue ring, which also marks the inner boundary of its extended disk. In the UV, GALEX images of both galaxies show spectacular structures, especially in the far-UV. Both resemble SR01, except that Malin 2 has a less well-defined inner ring, while the inner ring of UGC6614 is somewhat more regular than that of SR01.

The formation of giant LSBs has presented a puzzle ever since their discovery. Originally, giant LSBs were considered to represent spirals with forming disks (Bothun et al. 1987). Recent work breaks free of trying to fit all giant LSBs into an "unusual spiral" category. Studies of Malin 1 noted the presence of a smaller, relatively normal early-type disk (Barth 2007) with a normal inner rotation curve (Lelli et al. 2010). Previously, Quillen \& Pickering (1997) remarked on the extremely red disk colors of UGC6614 and Malin 2, suggestive of ETG populations. However, this connection with ETGs may not even hold for all giant LSBs. Of the 10 giant LSBs studied in the UV by Wyder et al. (2009) we notice that only Malin 1, Malin 2, and UGC6614 have central NUV $-r$ colors consistent with old populations. Furthermore, SDSS spectra of these three galaxies reveal high D4000 index values typical of ETGs and not found among normal spirals $(1.91,1.94$, and 1.77$) .{ }^{17}$ While strong emission lines are present in all three, the line ratios place them on the AGN branch of the BPT diagram, so no central SF is indicated. These data and our observations present an alternative

\footnotetext{
16 Giant LSBs are different from more common $d$ warf LSBs. The latter are diffuse, isolated dwarf galaxies, while the former are massive, have bulges, and feature more regular appearances, and it is only their disks that have low surface brightness.

17 From DR7 data processed by the MPA/JHU group.
} 
picture in which some giant LSBs are relatively normal S0 galaxies imbedded in extreme disks, possibly similar to the XUV disks of some spirals. The lack of optical blue light in SR01 and SR07 with respect to Malin 2 and UGC6614 may, however, indicate a more mature phase in the evolution of such "S0+LSBs." Donovan et al. (2009) in their study of the nearby S0 galaxy ESO 381-47 with a star-forming ring note that it may represent an ETG on its way to becoming a giant LSB. Interestingly, the far-UV morphology of ESO 381-47 observed by $G A L E X$ resembles that of SR07, with a main irregular ring surrounded by more diffuse and filamentary emission outside of it.

The connection between giant LSBs and S0s, or between LSBs and XUVs, does not by itself resolve the question of the source of star-forming gas. Noguchi (2001) presents numerical simulations which show that a normal high surface brightness disk can double its scale length due to secular processes involving bar formation in an unstable disk. An alternative model involving galaxy collisions (head-on mergers) was introduced by Mapelli et al. (2008). They propose that large disks are the aftermath of collisions that early on (100-200 Myr after the collision) appear as classical collisional rings (e.g., the Cartwheel galaxy) but later (0.5-1 Gyr after the collision) take on the form of extended disks. In both of these scenarios it is assumed that the gas is already present in the original, smaller disk. The "ruffled" UV appearance of SR01 and SR07 is strongly suggestive of interactions and less indicative of secular processes. However, the overall UV morphology of these two galaxies, as well as other "S0+LSBs," does not appear quite like the model predictions of Mapelli et al. (2008). Most notably, there is no evidence of the radial structures (spokes) that are seen in the simulations. On the other hand, the overall arclike structure and the inner irregular rings are features seen in more conventional simulations of minor mergers involving a disk galaxy and a companion on a parabolic orbit (Cox et al. 2008). These simulations generally show that minor mergers can lead to SF on galaxy-wide scales on the first passage, and not just in the centers as they undergo a final plunge. Perhaps under some circumstances such mergers can even lead to the very extended SF that are observed here.

\subsubsection{Tidal Arms and Pseudoarms}

The two galaxies in the UV arm class differ in appearance and likely have had different formation mechanisms. The arms in SR11 are part of a pseudoring, which is known from theoretical considerations to arise from a bar-induced resonance at the outer Lindblad resonance radius (Schwarz 1984; Athanassoula et al. 2009). The source of gas in these simulations is assumed to be internal. The presence of a full set of resonance features, and not just one wide ring as in the regular wide ring class, makes it more likely that the source of gas in SR11 is internal as well. There is one caveat to this. What is different between SR11 and most other nearby galaxies with pseudorings is that the latter are usually found in gas-rich late-type spirals (Buta \& Combes 1996). SR11, on the other hand, is dominated by old stellar populations, like the rest of our sample.

Of all the galaxies in our sample SR17 most clearly shows UV and optical signatures indicative of an interaction, with asymmetric arms that appear tidal in origin. The companion with which it is likely interacting is the galaxy to the upper right in the optical image (Figure 9). It is an S0 galaxy with no extended SF. Therefore, while the gas in SR17 is probably stirred to SF by the interaction, it does not seem to originate from the companion, but could instead be internal.

To summarize, both galaxies in this category may best fit the fading scenario.

\subsubsection{Summary of SF Mechanisms in ETGs with Extended SF}

Overall, the mechanisms that drive SF in ETGs with extended UV structures appear to be diverse. ETGs with regular wide rings probably form stars from the gas accreted relatively smoothly from the IGM, possibly in a prolonged quasi-static way. In ETGs with central holes the gas may be from the original blue cloud SF. ETGs in the narrow rings category may be acquiring their gas through various mechanisms including minor mergers. The merger scenario is perhaps more likely than accretion also for ETGs having irregular rings and large disks. Finally, the gas in the two ETGs with UV arms could again come from the original, internal gas supply that is not yet fully quenched. A tentative tally based on the analysis of each UV morphology group suggests that IGM accretion is the leading cause, being the likely mechanism in $55 \%$ of the ESF-ETG sample. As to the form of IGM accretion, we cannot exclude the quasistatic in which some accretion has been present ever since the major phase of SF ended, i.e., these galaxies may never have been entirely passive. In some $25 \%$ of the ESF-ETGs, the UV and optical morphologies and optical sizes are consistent with the fading of the original SF. In the remaining $20 \%$, nonaxisymmetric UV structures could result from the gas supplied by minor mergers. The relative scarcity of merger-induced SF is at odds with the findings of Kaviraj et al. (2009) who use numerical simulations to explain the entire population of starforming ETGs as resulting from minor mergers. We emphasize that our results apply to extended SF with little or no central SF, while minor merging would preferentially lead to nuclear SF. Therefore, the fraction of ETGs with SF that is due to mergers must be larger than the $20 \%$ that we find. In any case it is impossible for all SF to be explained in this way. We suggest that the result of Kaviraj et al. (2009) is critically sensitive to the timescale over which a minor merger leads to enhanced SFR. If the actual timescales are shorter by a factor of few than what their specific simulations predict $(\sim 2$ Gyr $)$, the merger mechanism would drop in significance from the dominant one to being a contributing mechanism.

Our results indicate that an external origin of gas is more common in our sample of ETGs with extended SF than an internal origin. However, we have to take into account that our selection misses $1 / 3$ of the ESF-ETG population with stronger central emission. If we assume that all of them are fading disks, the shares of ESF-ETGs with internal and external source of gas would become equal.

The fraction of ESF-ETGs that undergo fading of the original SF as opposed to subsequent "rejuvenation" could also be determined by measuring their neutral $\mathrm{H}$ gas content. Detection of $\mathrm{HI}$ reservoirs around $2 / 3$ of nearby field ETGs makes the external origin of gas a plausible scenario (Oosterloo et al. 2010). Further information is obtained by studying $\mathrm{H}$ I fractions. Fading galaxies would more likely be $\mathrm{H}$ I deficient (low specific H I mass, i.e., H I fraction). In contrast, rejuvenated ETGs (due to either the IGM accretion or merging) would have higher $\mathrm{H}_{\mathrm{I}}$ fractions, more similar to those of the blue cloud galaxies. A result pointing in the same direction as ours, that the green valley is being comprised of two distinct populations, was obtained by Cortese \& Hughes (2009) who showed that the nearby galaxies with normal $\mathrm{H}$ i content for their mass, yet in the green valley or 
on the UV-optical red sequence, frequently (6 out of 12 cases) have UV rings. They also show that part of this dichotomy is driven by environment-H I-deficient, and thus presumably fading, galaxies favor clusters and groups. As to the form of accretion (mergers versus smooth), Wang et al. (2011) using the GASS survey (Catinella et al. 2010) find no correlation between the $\mathrm{H}$ i fraction and the level of optical asymmetry, thus favoring smooth IGM accretion over satellite accretion.

\subsubsection{Small-scale Star-forming ETGs}

For six ETGs with small-scale star-forming regions, the situation is more straightforward. There the SF proceeds in smaller patches, thus excluding both fading and IGM accretion. If, as our estimate confirms, most of these patches are physically associated with the main optical source, then we are probably witnessing minor gas-rich mergers in various stages of coalescence. SR27 may be an early phase, with its companion being quite undisturbed. ${ }^{18}$ The companion looks more distorted in the UV in the case of SR30, and even more so in SR19. In SR13 the coalescence is almost complete. Finally, SR15 and 25 show central UV emission. Given their low mass, SR15 and SR25 could represent the local analogs of the high-redshift "blue spheroidals" of Im et al. (2001).

Because the optical images of galaxies in this class do not show much evidence of interaction, but simply look like superpositions of the main galaxy and the companion, these must be very minor mergers (e.g., with mass ratios greater than 10:1; Bournaud et al. 2007). Recall that our sample is selected against significant central SF, so a more complete exploration of the mechanisms of small-scale SF would be better addressed using different selections.

\subsection{Is Extended SF in ETGs an SO Phenomenon?}

None of the 19 galaxies with extended SF in our sample is an elliptical galaxy, and the analysis in Section 9 shows that the frequency of ETGs with extended UV excess follows trends that are consistent with it being a phenomenon that occurs only in S0s. Extended SF that are confidently known to be ellipticals appears to be rare even among the well-studied nearby galaxies. Curiously, we find only two such cases in the literature. One is Hoag's galaxy (type E0) with its detached star-forming ring for which Finkelman et al. (2011) recently argued for a formation through cold accretion. Another is an H I-rich NGC 5173 (type E1) with clumpy SF regions $\sim 4-10 \mathrm{kpc}$ from the center, possibly resulting from a merger (Vader \& Vigroux 1991). NGC 5173 is one of only two ellipticals with confident $\mathrm{CO}$ detection among the volume complete sample of 56 ellipticals within $42 \mathrm{Mpc}$ (Young et al. 2011). There is no evidence for SF in the GALEX FUV or NUV images of the other elliptical with CO detection (NGC 2768; Jeong et al. 2009). We tentatively show in Section 9 that even the central SF, expected from mergers, is also rarer in Es than in S0s. These results are corroborated by the wealth of information on the stellar populations in local ETGs that show that ellipticals do not have young populations (e.g., Kuntschner et al. 2010; Combes et al. 2007; Temi et al. 2009).

To understand why SF, and particularly extended SF, may be an exclusive S0 phenomenon in the context of external source of star-forming gas, it is important to understand why ETGs (particularly S0s) stop (or greatly reduce) forming stars in the first place. This question is the focus of many recent

\footnotetext{
18 Unless it is a chance superposition, see Section 4.2.
}

studies that try to explain the galaxy color bimodality and mechanisms behind it. An emerging consensus is that some feedback mechanism is required to quench the SF and bring the galaxy onto the red sequence, and that possibly another mechanism is required to keep the galaxy there, i.e., to maintain its quiescence by preventing the infall and/or cooling of fresh gas. Croton et al. (2006) suggest that a weak, radio-type AGN can serve this latter purpose. The need for such nonenvironmental, maintenance feedback is exacerbated by the existence of isolated quiescent galaxies (Croton \& Farrar 2008). One possibility then why ellipticals maintain their quiescence while many S0s do not could be that the former have more effective preventive feedback mechanisms. ${ }^{19}$ Considering that radio AGNs are primarily found in massive ellipticals this may not be surprising. Another, possibly related reason why ellipticals may be better at preventing SF, + is that many of them, especially the so-called slow rotators, have hot X-ray halos (whatever the heating mechanism is), which may prevent gas from cooling and forming stars (Nipoti \& Binney 2007; Sarzi et al. 2007; Kormendy et al. 2009).

A classification of ETGs into fast and slow rotators (Emsellem et al. 2007; Kormendy et al. 2009) was recently suggested as more fundamental than the S0 versus E classification (Emsellem et al. 2011). Considering that both the radio AGN and the X-ray gas are prevalent in slow rotators, it would be natural to expect the presence or absence of SF to follow this kinematical division rather than the S0 versus E division. The kinematic split appears to be corroborated by some recent results. For example, Shapiro et al. (2010) find that central SF (based on $8 \mu \mathrm{m}$ polycyclic aromatic hydrocarbon, $\mathrm{PAH}$, emission) is found only in fast rotators among the nearby SAURON galaxies. Similar absence of SF signatures (but in CO emission) among the slow rotators is seen for the larger ATLAS ${ }^{3 \mathrm{D}}$ sample (Young et al. 2011). Note, however, that basically all (94\%) of S0s are fast rotators, as well as most (66\%) Es (Cappellari et al. 2011), yet the CO detection rate is consistent with zero for all ellipticals in ATLAS ${ }^{3 \mathrm{D}}$, whether fast or slow. Shapiro et al. (2010) seemingly find two SAURON galaxies with PAH emission classified as ellipticals, but NGC 2974 is certainly not an E4 (RC3 classification), but rather an S0/a (Naim et al. 1995; Buta et al. 2010), while NGC 5845 is classified as an "uncertain E" because it contains a small stellar disk (Ebneter et al. 1988; Kormendy et al. 2005). Altogether, studies of nearby ETGs offer only sporadic evidence for $\mathrm{SF}$ in either slow- or fast-rotating ellipticals, suggesting that $\mathrm{SF}$ follows the traditional E versus SO divide.

In our study we see ample evidence for E versus S0 dichotomy when it comes to extended SF. The situation is less clear for small-scale SF, which may be present in some (perhaps less massive) ellipticals. In any case we believe that it is premature to abandon the classical distinction between Es and S0s in favor of the "new," kinematical one. In other words, calling fast rotating Es "misclassified lenticulars" (Cappellari et al. 2011) may not be fully justified since ellipticals, whether fast or slow, do not show evidence of extended SF, while many S0s of similar mass do.

The reason why some field S0s experience SF on the optical red sequence and others do not may be related to the absence, or the periodic nature of the maintenance feedback mechanisms (e.g., the AGN activity). In the first case the SF was quenched

\footnotetext{
19 Alternatively, in the fading scenario, some S0s have SF simply because they have not yet attained quiescence. Ellipticals, being typically older, would have acquired quiescence earlier in the cosmic history.
} 
during the transformation of a spiral into an S0 which involves the buildup of a bulge (e.g., "morphological quenching"; Martig et al. 2009), but no mechanism was in place to make the quiescence permanent. The second possibility (periodic preventive feedback) may be the result of intrinsically intermittent AGN activity in either Es or S0s. Gabor et al. (2011) showed that periodic AGN feedback provides better agreement between cosmological simulations and observations. While the periodic AGN activity would lead to periodic prevention of SFs in SOs, the "accumulation" of AGN energy in X-ray gas in ellipticals could render the SF prevention permanent (Kormendy et al. 2009).

One could argue that any elliptical that has experienced prolonged extended SF subsequent to its morphological transformation would re-grow a disk (e.g., Governato et al. 2009; De Lucia et al. 2011) and consequently be classified as an S0 (or later type) by definition. Whether such a scenario actually happens is by no means obvious because we do not seem to observe cases where the process of disk reformation is starting in or around ellipticals. Also, such a scenario would be conceivable only for fast-rotating ellipticals, as there are almost no slowrotating SOs. Second, since there are no S0s as massive as some ellipticals, this E to S0 transformation would still be restricted only to less massive ellipticals (i.e., on the less massive side of the "E-E" dichotomy; Kormendy et al. 2009).

The above discussion on the prevention of SF in Es as opposed to some SOs makes sense only if we assume that a galaxy is not completely detached from the supply of external gas. In the case of fading scenario, such detachment may be what is causing the galaxy to be fading in the first place, so considerations of preventive feedback are not needed.

\section{CONCLUSIONS}

This study explores the morphology of $z \sim 0.1$ optical red sequence galaxies (primarily early-type) with UV-detected SF. ETGs were selected to have strong UV excess yet weak central ionized emission. Such selection encompasses the large majority of ETGs with extended, galaxy-scale SF. Here are the main findings.

1. There are two modes of SF in our sample. The dominant one is extended on scales similar to or larger than the optical extent of a galaxy ( 19 of 25 , or $76 \%$ ). The secondary mode is one in which SF is concentrated in regions smaller than the optical extent. Such small-scale SF may represent cases in which the ETG assimilates a gas-rich dwarf. The remaining conclusions refer only to 19 ETGs with extended SF (ESF-ETGs).

2. None of the ESF-ETGs is optically classified as a true elliptical galaxy. All show the presence of stellar disks, usually of S0 type. Based on the analysis of the general SDSS/GALEX sample (for which Hubble types are not available), the incidence of extended SF is highest among the ETGs with $10.6<\log M_{*}<11.1$ and declines at both higher and lower masses; it also declines in ETGs with higher optical concentrations. These trends independently suggest that the extended SF is primarily a phenomenon of central (non-satellite) S0 galaxies. Tentatively, extended SF is estimated to be present in no more than $2 \%$ of massive ellipticals (with fraction closer to zero not being excluded), as opposed to $\sim 20 \%$ for S0s. The latter is similar to the incidence rate of XUV disks in spirals, hinting at similar fueling mechanism(s).
3. In all but one case the SF in ESF-ETGs takes place in UV rings with diameters of tens of kpc. The star-forming rings are not conspicuous in the optical (except one) and less than a half of hosts have an optical stellar bar. The latter indicates that the UV rings are not necessarily maintained by bar resonances. The dominance of UV rings is partially due to our selection criteria. However, even if we allow all ETGs with extended SF not covered by our selection to have non-ring morphology (e.g., in-filled disks), the UV ring incidence would still dominate $(\sim 2 / 3)$. The morphology of UV rings varies and may indicate different sources of star-forming gas or modes of SF.

4. The morphological analysis suggests that the recent or ongoing IGM accretion is the likeliest dominant mechanism for the source of gas in ESF-ETGs having wide UV rings, while the fading of the original SF (the latest stage of quenching) may be responsible for SF in disks with central UV holes. The latter also show signatures of optically fading spiral structure. Galaxy interactions may fuel SF in several other cases, including the two extreme disks (diameters $\sim 70 \mathrm{kpc}$ ), but in general the disturbances are not visible in the optical but only in the UV, suggesting at most very minor mergers. The ESF-ETGs with extreme disks resemble the giant LSB galaxies Malin 2 and UGC6614 and together with them may represent a distinct class of giant LSBs, which we call S0+LSBs.

5. Analysis of disk sizes shows that in roughly half of the ESF-ETGs the optical disks are $\sim 50 \%$ larger than in the underlying population of quiescent ETGs of the same mass, suggesting that significant disk buildup occurred in ESFETGs after the galaxy concluded the original epoch of SF. Such disk enhancement is consistent with long-lasting and relatively smooth accretion from the IGM and not the more recent merger-supplied gas. ESF-ETGs, whether their disks have been enhanced in the optical or not, on average have a larger ratio of UV to optical size than the comparable green valley galaxies, suggesting that the source of gas is more likely to be external (assuming that most green valley galaxies are fading onto the red sequence due quenching that cuts off the infalling gas).

6. Altogether the IGM accretion may be responsible for SF in $55 \%$ of the sample, followed by fading of the original SF in $25 \%$ and minor mergers in the remaining $20 \%$. External origin of gas (from accretion and minor mergers) thus together accounts for $3 / 4$ of the sample. The IGM accretion in these galaxies may have been present ever since the major SF ended, i.e., these galaxies may never have been truly passive but are instead suspended in the green valley (quasi-static IGM accretion). This is consistent with the fact that the plane of new SF coincides with the old disk.

The results at which we arrive here point to a range of mechanisms driving SF in ETGs. Keeping in mind that our sample does not include filled-in disks, we find that the extended SF in most (but not all) cases looks like it is the result of a gradual, non-merger process. This is in general agreement with the results presented in Paper II, where it is shown that UV and optical colors do not favor bursty SF for most of our sample. Our conclusions primarily hold for extended SF. Small-scale central or circumnuclear SF, which our selection disfavors but could be as frequent as the extended SF, might instead preferentially be the result of minor mergers.

The widespread occurrence of galaxy-scale SF in ETGs reported in this paper may appear to contrast the results from the 
SAURON sample, in which the extended SF is reported for only 2 out of 48 galaxies (NGC 2974 and NGC 2685). The primary reason behind this is in SAURON sample selection-which is $1 / 2$ S0s and $1 / 2$ ellipticals by design, with a disproportionate fraction being cluster galaxies. When taken into account that the extended SF is common only in field S0s, the small numbers of extended SF in the SAURON sample are not surprising. The incidence of galaxy-wide SF will certainly be higher in the ATLAS $^{3 \mathrm{D}}$ sample, which includes all ETGs within $42 \mathrm{Mpc}$, but even then it will be the UV, rather than the emission line maps, that will have the requisite surface brightness sensitivity to detect low levels of SF.

The absence of true ellipticals from our sample of ESF-ETGs is intriguing and underlines the importance of distinguishing between ellipticals and S0s, especially when considering SF. Results from other studies seem to support this: ellipticals of intermediate and high mass, whether rotating fast or slow, do not show strong evidence of either extended or central current SF. The traditional view of all non-dwarf ellipticals as being "red and dead" is therefore basically upheld even in field populations. In contrast, many (perhaps even the majority, if the process is episodic) field S0s maintain or reacquire some level of SF activity.

S.S. thanks Ronald Buta, T. J. Cox, Alister Graham, Janice C. Lee, and Antonietta Marino for useful discussions and comments, and Aaron Barth for help regarding his excellent ATV.pro image display tool. S.S. also acknowledges observing assistance provided by WIYN/KPNO staff and Steven Janowiecki. Special thanks to Karim Salim for producing Figure 20. J.J.F. acknowledges financial support from a UCSC Regent's Fellowship and HST grant GO-11175. Based on observations made with the NASA/ESA Hubble Space Telescope under program GO-11158.

\section{APPENDIX}

\section{SAMPLE SELECTION AND THE POPULATION OF ETGs WITH EXTENDED SF}

As discussed in Section 2 the original selection criteria to obtain the HST sample were geared toward ETGs with no evidence for SF based on spectroscopic criteria. In contrast, the sample exhibits widespread SF. This extended SF is the focus of the present work. However, since the original selection criteria were not optimized to select a full population of ETGs with extended SF, it may have inadvertently excluded those that also have significant SF in the central $(\sim 5 \mathrm{kpc})$ regions. Therefore, it is important to establish what fraction of ETGs with extended SF are not centrally quiescent and therefore not probed by our sample. Such galaxies may have significantly different morphology than the galaxies we selected which could affect the tally of different star-forming processes that we explore.

To probe red sequence ESF-ETGs in a completely unbiased way we would need to replace the spectroscopic quiescence criterion (number 5 in Section 2) with a criterion that ensures integrated (global) red optical colors and a criterion that selects only extended SF. Optical red color did not feature as the original selection criterion and yet the sample galaxies were all red. The reason behind this can be seen in Figure 21 where we plot the global optical color $g-r$ versus the specific SFR within the SDSS fiber $\left(3^{\prime \prime}\right)$. The underlying population (shown as gray scale) are galaxies with the mass and redshift range of the HST sample $\left(10.2<\log M_{*}<11.2,0.08<z<0.12\right)$,

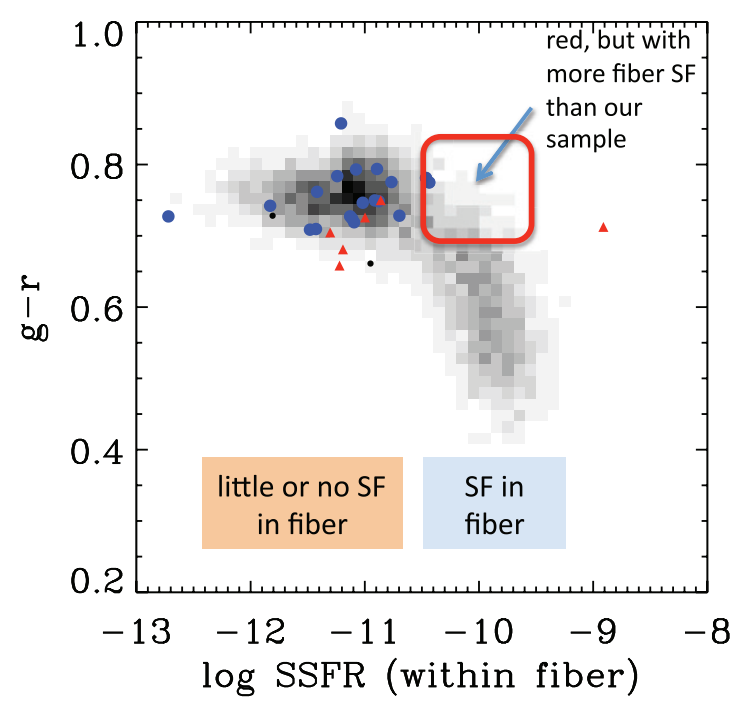

Figure 21. Relation between the global optical color ( $g-r, K$-corrected) and the star formation activity within the SDSS fiber that samples the inner $\sim 5 \mathrm{kpc}$ region of a galaxy. Fiber specific SFRs are obtained from SDSS DR4 MPA/JHU catalogs. Underlying population (gray scale) has the redshift and mass range of the HST sample (symbols) and omits edge-on (reddened) galaxies. Galaxies with low SF in fiber also tend to be globally red, but some red sequence galaxies can have higher fiber specific SFR than probed by our sample (red rectangle).

(A color version of this figure is available in the online journal.)

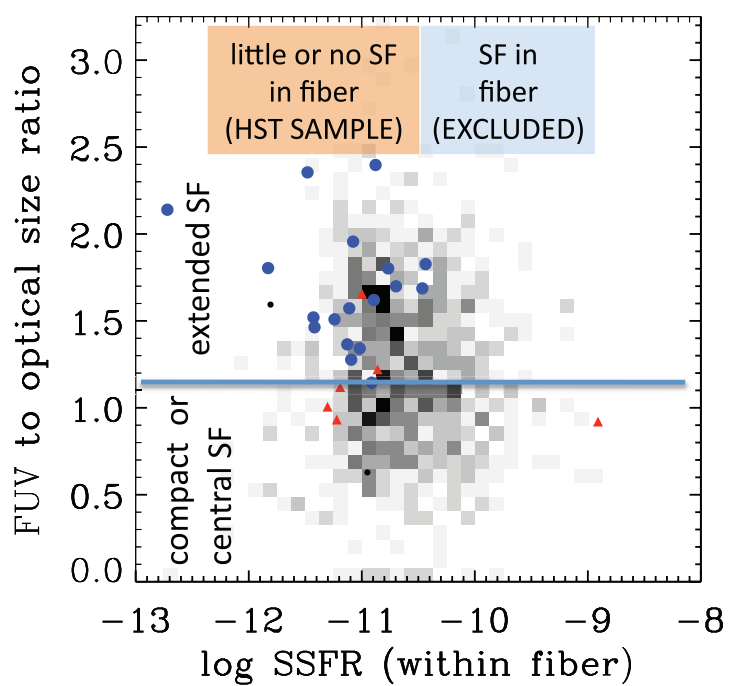

Figure 22. Level of the extendedness of SF (the FUV to optical size ratio) against the fiber specific SFR. ETGs with extended SF tend to be above size ratio of 1.15 (horizontal line). The $H S T$ sample probes extended SF in ETGs with lower fiber specific SFR, which represents $2 / 3$ of the population.

(A color version of this figure is available in the online journal.)

with the edge-on $(b / a>0.5)$ presumably dusty disks removed. We see that galaxies with little or no SF within the fiber $\left(\log \mathrm{SFR} / M_{*} \lesssim-10.5\right)$ are invariably red $(g-r>0.7)$, which explains why our HST sample (symbols) and especially the ETGs with extended SF (blue dots) are on the red sequence). Optically blue galaxies also tend to have higher specific SFRs within the fiber, but there do exist some galaxies which are on the red sequence, but whose fiber SF is higher than the low levels allowed by our original spectroscopic criterion.

To see what fraction of red sequence ETGs have high fiber SF and are extended in the UV, we show UV to optical size ratio against the specific SFR in the fiber, but now only of red $(g-r>0.7)$ ETGs $(C>2.5)$, and again without highly 
inclined systems (Figure 22). The information on UV size comes from GALEX and is therefore not very precise, but as discussed in Section 7 it allows selection of ETGs with extended SF. The HST sample of ESF-ETGs (blue dots) has size ratios $>1.15$ and fiber specific SFRs of $\log \mathrm{SFR} / M_{*}<-10.5$. There are 296 underlying ETGs with characteristics like that and, therefore, likely similar morphologies as the HST sample. On the other hand, there are 157 ETGs which are also extended (size ratio $>1.15$ ) but with higher fiber specific SFRs. This represents $35 \%$ of all ESF-ETGs. If all of these ESF-ETGs had very different morphologies from centrally quiescent ESF-ETGs, then certain morphologies (e.g., infilled disks) would be underrepresented in our sample in favor of others (e.g., rings), which may affect the relative importance of the various SF mechanisms that we discuss.

To summarize, at least 2/3 of ETGs with extended SF likely have similar UV morphologies to our ESF-ETGs, while only up to $1 / 3$ may be different. Thus, our conclusions are relevant for the majority of the ETG population with ESF.

\section{REFERENCES}

Aguerri, J. A. L., Méndez-Abreu, J., \& Corsini, E. M. 2009, A\&A, 495, 491 Athanassoula, E., Romero-Gómez, M., \& Masdemont, J. J. 2009, MNRAS, 394, 67

Baillard, A., Bertin, E., de Lapparent, V., et al. 2011, A\&A, 532, A74

Barnes, J. E., \& Hernquist, L. 1996, ApJ, 471, 115

Barth, A. J. 2007, AJ, 133, 1085

Bauermeister, A., Blitz, L., \& Ma, C.-P. 2010, ApJ, 717, 323

Bekki, K. 2009, MNRAS, 399, 2221

Bernardi, M., Shankar, F., Hyde, J. B., et al. 2010, MNRAS, 404, 2087

Bernardi, M., Sheth, R. K., Annis, J., et al. 2003, AJ, 125, 1817

Blanton, M. R., \& Roweis, S. 2007, AJ, 133, 734

Bothun, G. D., Impey, C. D., Malin, D. F., \& Mould, J. R. 1987, AJ, 94, 23

Bournaud, F., Jog, C. J., \& Combes, F. 2007, A\&A, 476, 1179

Brinchmann, J., Charlot, S., White, S. D. M., et al. 2004, MNRAS, 351, 1151

Buta, R., \& Combes, F. 1996, Fundam. Cosm. Phys., 17, 95

Buta, R., \& Crocker, D. A. 1991, AJ, 102, 1715

Buta, R. J. 2011, arXiv:1102.0550

Buta, R. J., Sheth, K., Regan, M., et al. 2010, ApJS, 190, 147

Cappellari, M., Emsellem, E., Krajnović, D., et al. 2011, MNRAS, 416, 1680

Catinella, B., Schiminovich, D., Kauffmann, G., et al. 2010, MNRAS, 403, 683

Cheng, J. Y., Faber, S. M., Simard, L., et al. 2011, MNRAS, 412, 727

Combes, F. 2006, EAS Pub. Ser., 20, 97

Combes, F., Young, L. M., \& Bureau, M. 2007, MNRAS, 377, 1795

Cortese, L., \& Hughes, T. M. 2009, MNRAS, 400, 1225

Crocker, A. F., Bureau, M., Young, L. M., \& Combes, F. 2011, MNRAS, 410 , 1197

Croton, D. J., \& Farrar, G. R. 2008, MNRAS, 386, 2285

Croton, D. J., Springel, V., White, S. D. M., et al. 2006, MNRAS, 365, 11

Cox, C. 2009, Instrument Science Report ACS 2009-02, 2, 7 pp

Cox, T. J., Jonsson, P., Somerville, R. S., Primack, J. R., \& Dekel, A. 2008, MNRAS, 384, 386

Davé, R., Oppenheimer, B. D., \& Finlator, K. 2011, MNRAS, 415, 11

Dekel, A., \& Birnboim, Y. 2006, MNRAS, 368, 2

De Lucia, G., Fontanot, F., Wilman, D., \& Monaco, P. 2011, MNRAS, 414, 1439

De Lucia, G., Springel, V., White, S. D. M., Croton, D., \& Kauffmann, G. 2006, MNRAS, 366, 499

Donas, J., Deharveng, J.-M., Rich, R. M., et al. 2007, ApJS, 173, 597

Donovan, J. L., Serra, P., van Gorkom, J. H., et al. 2009, AJ, 137, 5037

Dorman, B., O'Connell, R. W., \& Rood, R. T. 2003, ApJ, 591, 878

Drory, N., \& Fisher, D. B. 2007, ApJ, 664, 640

Ebneter, K., Davis, M., \& Djorgovski, S. 1988, AJ, 95, 422

Elmegreen, D. M., Elmegreen, B. G., Combes, F., \& Bellin, A. D. 1992, A\&A 257,17

Emsellem, E., Cappellari, M., Krajnović, D., et al. 2007, MNRAS, 379, 401

Emsellem, E., Cappellari, M., Krajnović, D., et al. 2011, MNRAS, 414, 888

Fang, J. J., Faber, S. M., Salim, S., Graves, G. J., \& Rich, R. M. 2012, ApJ, submitted

Finkelman, I., Moiseev, A., Brosch, N., \& Katkov, I. 2011, MNRAS, 418, 1834

Fisher, D. B. 2006, ApJ, 642, L17
Gabor, J. M., Davé, R., Oppenheimer, B. D., \& Finlator, K. 2011, MNRAS, 417, 2676

Gil de Paz, A., Boissier, S., Madore, B. F., et al. 2007, ApJS, 173, 185

Governato, F., Brook, C. B., Brooks, A. M., et al. 2009, MNRAS, 398, 312

Hao, J., McKay, T. A., Koester, B. P., et al. 2010, ApJS, 191, 254

Hawarden, T. G., van Woerden, H., Goss, W. M., Mebold, U., \& Peterson, B. A. 1979, A\&A, 76, 230

Im, M., Faber, S. M., Gebhardt, K., et al. 2001, AJ, 122, 750

Jeong, H., Bureau, M., Yi, S. K., Krajnović, D., \& Davies, R. L. 2007, MNRAS, 376, 1021

Jeong, H., Yi, S. K., Bureau, M., et al. 2009, MNRAS, 398, 2028

Kannappan, S. J., Guie, J. M., \& Baker, A. J. 2009, AJ, 138, 579

Kauffmann, G., Charlot, S., \& White, S. D. M. 1996, MNRAS, 283, L117

Kauffmann, G., Heckman, T. M., Budavári, T., et al. 2007, ApJS, 173, 357

Kaviraj, S. 2010, MNRAS, 406, 382

Kaviraj, S., Peirani, S., Khochfar, S., Silk, J., \& Kay, S. 2009, MNRAS, 394 1713

Kelson, D. D., \& Holden, B. P. 2010, ApJ, 713, L28

Kennicutt, R. C., Jr., Tamblyn, P., \& Congdon, C. E. 1994, ApJ, 435, 22

Kereš, D., \& Hernquist, L. 2009, ApJ, 700, L1

Kereš, D., Katz, N., Weinberg, D. H., \& Davé, R. 2005, MNRAS, 363, 2

Koekemoer, A. M., Fruchter, A. S., Hook, R. N., \& Hack, W. 2002, in HST Calibration Workshop, Hubble after the Installation of the ACS and the NICMOS Cooling System, ed. S. Arribas et al. (Baltimore, MD: STScI), 337

Kormendy, J., Fisher, D. B., Cornell, M. E., \& Bender, R. 2009, ApJS, 182, 216

Kormendy, J., Gebhardt, K., Fisher, D. B., et al. 2005, AJ, 129, 2636

Krumholz, M. R., \& McKee, C. F. 2008, Nature, 451, 1082

Kuntschner, H., Emsellem, E., Bacon, R., et al. 2010, MNRAS, 408, 97

Lackner, C. N., \& Gunn, J. E. 2012, MNRAS, 421, 2277

Larson, R. B., Tinsley, B. M., \& Caldwell, C. N. 1980, ApJ, 237, 692

Lee, J. C., Gil de Paz, A., Tremonti, C., et al. 2009, ApJ, 706, 599

Leitner, S. N., \& Kravtsov, A. V. 2011, ApJ, 734, 48

Lelli, F., Fraternali, F., \& Sancisi, R. 2010, A\&A, 516, A11

Lemonias, J. J., Schiminovich, D., Thilker, D., et al. 2011, ApJ, 733, 74

Lotz, J. M., Jonsson, P., Cox, T. J., et al. 2011, ApJ, 742, 103

Lupton, R., Blanton, M. R., Fekete, G., et al. 2004, PASP, 116, 133

Macchetto, F., Pastoriza, M., Caon, N., et al. 1996, A\&AS, 120, 463

Maller, A. H., Katz, N., Kereš, D., Davé, R., \& Weinberg, D. H. 2006, ApJ, 647, 763

Mandelbaum, R., Seljak, U., Kauffmann, G., Hirata, C. M., \& Brinkmann, J. 2006, MNRAS, 368, 715

Mapelli, M., Moore, B., Ripamonti, E., et al. 2008, MNRAS, 383, 1223

Marino, A., Rampazzo, R., Bianchi, L., et al. 2011, MNRAS, 411, 311

Martig, M., Bournaud, F., Teyssier, R., \& Dekel, A. 2009, ApJ, 707, 250

Martin, D. C., Fanson, J., Schiminovich, D., et al. 2005, ApJ, 619, L1

Martin, D. C., Wyder, T. K., Schiminovich, D., et al. 2007, ApJS, 173, 342

Masters, K. L., Mosleh, M., Romer, A. K., et al. 2010a, MNRAS, 405, 783

Masters, K. L., Nichol, R., Bamford, S., et al. 2010b, MNRAS, 404, 792

Maybhate, A., et al. 2010, ACS Instrument Handbook, Version 9.0 (Baltimore, MD: STScI)

Mihos, J. C., \& Hernquist, L. 1994, ApJ, 425, L13

Moffett, A. J., Kannappan, S. J., Baker, A. J., \& Laine, S. 2012, ApJ, 745, 34

Naim, A., Lahav, O., Buta, R. J., et al. 1995, MNRAS, 274, 1107

Nipoti, C., \& Binney, J. 2007, MNRAS, 382, 1481

Noguchi, M. 2001, MNRAS, 328, 353

Oosterloo, T., Morganti, R., Crocker, A., et al. 2010, MNRAS, 409, 500

Peirani, S., Crockett, R. M., Geen, S., et al. 2010, MNRAS, 405, 2327

Quillen, A. C., \& Pickering, T. E. 1997, arXiv:astro-ph/9705115

Rich, R. M., Salim, S., Brinchmann, J., et al. 2005, ApJ, 619, L107

Salim, S., Dickinson, M., Michael Rich, R., et al. 2009, ApJ, 700, 161

Salim, S., \& Rich, R. M. 2010, ApJ, 714, L290 (SR2010)

Salim, S., Rich, R. M., Charlot, S., et al. 2007, ApJS, 173, 267

Sarzi, M., Bacon, R., Cappellari, M., et al. 2007, New Astron. Rev., 51, 18

Sarzi, M., Shields, J. C., Schawinski, K., et al. 2010, MNRAS, 402, 2187

Schawinski, K., Dowlin, N., Thomas, D., Urry, C. M., \& Edmondson, E. 2010, ApJ, 714, L108

Schawinski, K., Lintott, C., Thomas, D., et al. 2009, MNRAS, 396, 818

Schwarz, M. P. 1984, Proc. Astron. Soc. Aust., 5, 464

Schweizer, F., Ford, W. K., Jr., Jedrzejewski, R., \& Giovanelli, R. 1987, ApJ, 320,454

Shapiro, K. L., Falcón-Barroso, J., van de Ven, G., et al. 2010, MNRAS, 402, 2140

Smith, R. J., Lucey, J. R., \& Carter, D. 2012, MNRAS, 421, 2982

Springel, V., Di Matteo, T., \& Hernquist, L. 2005, MNRAS, 361, 776

Strateva, I., Ivezić, Ž., Knapp, G. R., et al. 2001, AJ, 122, 1861

Temi, P., Brighenti, F., \& Mathews, W. G. 2009, ApJ, 695, 1 
Teplitz, H. I., Siana, B., Brown, T. M., et al. 2006, AJ, 132, 853

Thilker, D. A., Bianchi, L., Meurer, G., et al. 2007, ApJS, 173, 538

Thilker, D. A., Bianchi, L., Schiminovich, D., et al. 2010, ApJ, 714, L171

Trager, S. C., Faber, S. M., Worthey, G., \& González, J. J. 2000, AJ, 120, 165

Vader, J. P., \& Vigroux, L. 1991, A\&A, 246, 32

van Dokkum, P. G. 2001, PASP, 113, 1420

Vulcani, B., Poggianti, B. M., Dressler, A., et al. 2011, MNRAS, 413, 921

Wang, J., Kauffmann, G., Overzier, R., et al. 2011, MNRAS, 412, 1081
Wardle, M., \& Knapp, G. R. 1986, AJ, 91, 23

Weijmans, A.-M., Krajnović, D., van de Ven, G., et al. 2008, MNRAS, 383, 1343

Wyder, T. K., Martin, D. C., Barlow, T. A., et al. 2009, ApJ, 696, 1834

Wyder, T. K., Martin, D. C., Schiminovich, D., et al. 2007, ApJS, 173, 293

Xu, C. K., Donas, J., Arnouts, S., et al. 2005, ApJ, 619, L11

Yi, S. K., Yoon, S.-J., Kaviraj, S., et al. 2005, ApJ, 619, L111

Young, L. M., Bureau, M., Davis, T. A., et al. 2011, MNRAS, 414, 940

Young, J. S., \& Scoville, N. 1982, ApJ, 260, L41 